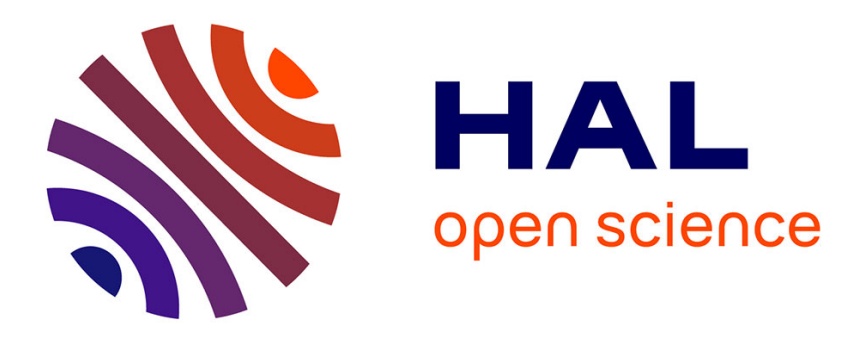

\title{
A 5-wave relaxation solver for the shallow water MHD system
}

François Bouchut, Xavier Lhébrard

\section{To cite this version:}

François Bouchut, Xavier Lhébrard. A 5-wave relaxation solver for the shallow water MHD system. Journal of Scientific Computing, 2016, 68 (1), pp.92-115. 10.1007/s10915-015-0130-4 . hal$01131293 \mathrm{v} 2$

\section{HAL Id: hal-01131293 \\ https://hal.science/hal-01131293v2}

Submitted on 13 Jun 2016

HAL is a multi-disciplinary open access archive for the deposit and dissemination of scientific research documents, whether they are published or not. The documents may come from teaching and research institutions in France or abroad, or from public or private research centers.
L'archive ouverte pluridisciplinaire HAL, est destinée au dépôt et à la diffusion de documents scientifiques de niveau recherche, publiés ou non, émanant des établissements d'enseignement et de recherche français ou étrangers, des laboratoires publics ou privés. 


\title{
A 5-wave relaxation solver for the shallow water MHD system
}

\author{
François Bouchut* Xavier Lhébrard*
}

\begin{abstract}
The shallow water magnetohydrodynamic system describes the thin layer evolution of the solar tachocline. It is obtained from the three dimensional incompressible magnetohydrodynamic system similarly as the classical shallow water system is obtained from the incompressible NavierStokes equations. The system is hyperbolic and has two additional waves with respect to the shallow water system, the Alfven waves. These are linearly degenerate, and thus do not generate dissipation. In the present work we introduce a 5-wave approximate Riemann solver for the shallow water magnetohydrodynamic system, that has the property to be non dissipative on Alfven waves. It is obtained by solving a relaxation system of Suliciu type, and is similar to HLLC type solvers. The solver is positive and entropy satisfying, ensuring its robustness. It has sharp wave speeds, and does not involve any iterative procedure.
\end{abstract}

Keywords: Shallow water magnetohydrodynamics, approximate Riemann solver, relaxation, contact discontinuities, entropy inequality

Mathematics Subject Classification: 76W05, 76M12, 35L65

\section{Introduction}

The shallow water magnetohydrodynamic (SWMHD) system has been introduced in [18] to describe the thin layer evolution of the solar tachocline. It is written in $2 \mathrm{~d}$ in the tangent plane approximation as

$$
\begin{gathered}
\partial_{t} h+\nabla \cdot(h \mathbf{u})=0, \\
\partial_{t}(h \mathbf{u})+\nabla \cdot(h \mathbf{u} \otimes \mathbf{u}-h \mathbf{b} \otimes \mathbf{b})+\nabla\left(g h^{2} / 2\right)=0, \\
\partial_{t}(h \mathbf{b})+\nabla \cdot(h \mathbf{b} \otimes \mathbf{u}-h \mathbf{u} \otimes \mathbf{b})+\mathbf{u} \nabla \cdot(h \mathbf{b})=0,
\end{gathered}
$$

where $g>0$ is the gravity constant, $h \geq 0$ is the thickness of the fluid, $\mathbf{u}=(u, v)$ is the velocity, $\mathbf{b}=(a, b)$ is the magnetic field, and the notation $\nabla \cdot(\mathbf{b} \otimes \mathbf{u})$ is for the vector with index $i$ given by $\sum_{j} \partial_{j}\left(b_{i} u_{j}\right)$. The system should be complemented with Coriolis force and topography, but these sources will not be considered in this paper.

\footnotetext{
*Université Paris-Est, Laboratoire d'Analyse et de Mathématiques Appliquées (UMR 8050), CNRS, UPEM, UPEC, F-77454, Marne-la-Vallée, France (Francois.Bouchut@upem.fr), (Xavier.Lhebrard@u-pem.fr)
} 
The system (1.1)-(1.3) is endowed with an entropy (energy) inequality

$$
\begin{aligned}
& \partial_{t}\left(\frac{1}{2} h|\mathbf{u}|^{2}+\frac{1}{2} g h^{2}+\frac{1}{2} h|\mathbf{b}|^{2}\right) \\
& +\nabla \cdot\left(\left(\frac{1}{2} h|\mathbf{u}|^{2}+g h^{2}+\frac{1}{2} h|\mathbf{b}|^{2}\right) \mathbf{u}-h \mathbf{b}(\mathbf{b} \cdot \mathbf{u})\right) \leq 0,
\end{aligned}
$$

that becomes an equality in the absence of shocks.

The induction equation (1.3) implies, by taking its divergence, that

$$
\partial_{t}(\nabla \cdot(h \mathbf{b}))+\nabla \cdot(\mathbf{u} \nabla \cdot(h \mathbf{b}))=0
$$

meaning that $\nabla \cdot(h \mathbf{b})$ is transported at velocity $\mathbf{u}$. In particular, $\nabla \cdot(h \mathbf{b})$ remains identically zero if it vanishes initially. This situation $\nabla \cdot(h \mathbf{b})=0$ (that cancels the last term in (1.3)) is indeed the physically relevant one, but for numerical purposes it is convenient to relax this constraint and consider general data. The particular form (1.3) has been introduced in [13] for the SWMHD, and in [20] for the full MHD system. It enables to use one-dimensional solvers in two dimensions, indeed this is why the term $\mathbf{u} \nabla \cdot(h \mathbf{b})$ has been added in (1.3).

Multidimensional simulations of the SWMHD system have been performed in $[21,22]$. The system is closely related to the MHD system, to which many works have been devoted. An important issue in multidimensional simulations is to minimize the numerical viscosity by using accurate solvers, in particular on contact discontinuities; while being robust, see for example [16, 25, 1].

If dependency is only in one spatial variable $x$, the system simplifies to

$$
\begin{aligned}
\partial_{t} h+\partial_{x}(h u) & =0, \\
\partial_{t}(h u)+\partial_{x}\left(h u^{2}+P\right) & =0, \\
\partial_{t}(h v)+\partial_{x}\left(h u v+P_{\perp}\right) & =0, \\
\partial_{t}(h a)+u \partial_{x}(h a) & =0, \\
\partial_{t}(h b)+\partial_{x}(h b u-h a v)+v \partial_{x}(h a) & =0,
\end{aligned}
$$

with

$$
P=g \frac{h^{2}}{2}-h a^{2}, \quad P_{\perp}=-h a b,
$$

and the energy inequality (1.4) becomes

$$
\begin{aligned}
& \partial_{t}\left(\frac{1}{2} h\left(u^{2}+v^{2}\right)+\frac{1}{2} g h^{2}+\frac{1}{2} h\left(a^{2}+b^{2}\right)\right) \\
& +\partial_{x}\left(\left(\frac{1}{2} h\left(u^{2}+v^{2}\right)+g h^{2}+\frac{1}{2} h\left(a^{2}+b^{2}\right)\right) u-h a(a u+b v)\right) \leq 0 .
\end{aligned}
$$

The eigenvalues of the system (1.6)-(1.10) are $u, u \pm|a|, u \pm \sqrt{a^{2}+g h}$. The associated waves are called respectively material (or divergence) waves, Alfven waves and magnetogravity waves, see $[15,26]$. Some of these waves will have the same speed when $a$ or $h$ vanishes, hence the system is nonstrictly hyperbolic.

The system has three types of contact discontinuities corresponding to linearly degenerate eigenvalues: the material contacts associated to the eigenvalue 
$u$, the left Alfven contacts associated to $u-|a|$, and the right Alfven contacts associated to $u+|a|$. The jump relations associated to these contact discontinuities are as follows. Across a material contact, the quantities $u, v, g \frac{h^{2}}{2}-h a^{2}, h a b$ are constant. Across an Alfven contact, the quantities $h, u, a$ are constant, and moreover for a left Alfven contact $b \operatorname{sgn} a-v$ is constant, while for a right Alfven contact $b \operatorname{sgn} a+v$ is constant.

The system (1.6)-(1.10) is nonconservative in the variables $h a, h b$. However, $h a$ jumps only through the material contacts, where $u$ and $v$ are continuous. Therefore, there is indeed no ambiguity in the non conservative products $u \partial_{x}(h a)$ and $v \partial_{x}(h a)$, that are well-defined.

A finite volume scheme for the quasilinear system (1.6)-(1.10) can be classically built following Godunov's approach, considering piecewise constant approximations of

$$
U=(h, h u, h v, h a, h b),
$$

and invoking an approximate Riemann solver at the interface between two cells, see for example [19] or [6, Section 2.3]. A difficulty is however that the system is not conservative. The energy is nevertheless obviously convex with respect to $U$. The SWMHD system is closely related to the compressible MHD system, for which several entropy schemes are known $[17,4,14,9]$. In this paper we apply the relaxation approach of $[5,6,8,12]$ to the SWMHD system, in order to get an approximate Riemann solver that is entropy satisfying, ensuring robustness, while being exact on isolated Alfven contacts. The relaxation system is of Suliciu type as introduced in [24], and the approximate Riemann solver belongs to the family of HLLC solvers, as in [19, 6, 2, 23, 3, 16, 1].

The paper is organized as follows. In Section 2 we describe the relaxation approximate solver and its entropy property. In Section 3 we derive explicit optimal choices of the speeds that enable to obtain stability and accuracy. In Section 4 we state our main theorem giving the properties of our relaxation approximate Riemann solver. Finally, in Section 5 we perform numerical tests.

\section{Approximate Riemann solver}

\subsection{Relaxation approach}

In order to get an approximate Riemann solver for (1.6)-(1.10), we use a standard relaxation approach, used for example in [6] for the Euler equations, in [9] for the MHD equations and in [7] for shallow elastic fluids. An abstract general description can be found in [12], and related works are [10,11]. The approach enables to naturally handle the energy inequality (1.12), and also preserves the positivity of density. Its structure has however to be well-chosen in order to resolve exactly isolated Alfven contacts.

We introduce new variables $\pi, \pi_{\perp}$, the relaxed pressures, and $c_{a}, c$ intended 
to parametrize the speeds. The form of the relaxation system is as follows,

$$
\begin{aligned}
\partial_{t} h+\partial_{x}(h u) & =0, \\
\partial_{t}(h u)+\partial_{x}\left(h u^{2}+\pi\right) & =0, \\
\partial_{t}(h v)+\partial_{x}\left(h u v+\pi_{\perp}\right) & =0, \\
\partial_{t}(h a)+u \partial_{x}(h a) & =0, \\
\partial_{t}(h b)+\partial_{x}(h b u-h a v)+v \partial_{x}(h a) & =0, \\
\partial_{t}(h \pi)+\partial_{x}(h \pi u)+c^{2} \partial_{x} u & =0, \\
\partial_{t}\left(h \pi_{\perp}\right)+\partial_{x}\left(h \pi_{\perp} u\right)+c_{a}^{2} \partial_{x} v & =0, \\
\partial_{t} c+u \partial_{x} c & =0, \\
\partial_{t} c_{a}+u \partial_{x} c_{a} & =0 .
\end{aligned}
$$

The approximate Riemann solver can be defined as follows, starting from left and right values $U_{l}, U_{r}$ at an interface.

- Solve the Riemann problem for (2.1)-(2.9) with initial data obtained by completing $U_{l}, U_{r}$ by the equilibrium relations

$$
\begin{aligned}
& \pi_{l}=P_{l} \equiv\left(g h^{2} / 2-h a^{2}\right)_{l}, \\
& \pi_{r}=P_{r} \equiv\left(g h^{2} / 2-h a^{2}\right)_{r}, \\
& \left(\pi_{\perp}\right)_{l}=\left(P_{\perp}\right)_{l} \equiv(-h a b)_{l}, \\
& \left(\pi_{\perp}\right)_{r}=\left(P_{\perp}\right)_{r} \equiv(-h a b)_{r},
\end{aligned}
$$

and with suitable positive values of $c_{l}, c_{r}, c_{a, l}, c_{a, r}$ that will be discussed further on, essentially in Section 3.

- Retain in the solution only the variables $h, h u, h v, h a, h b$. The result is a vector called $R\left(x / t, U_{l}, U_{r}\right)$.

We can remark that the relaxation system (2.1)-(2.9) is identical to the 5 -wave relaxation system in $[9$, equations (5.5)-(5.7) with $b=0]$, with the identification $B_{x}=h a, B_{\perp}=h b$. However, the initialization (2.10) differs from that of the MHD equations, [9, equation (2.10)]. Indeed, the homogeneity in magnetic terms is different in the SWMHD and MHD systems.

Intuitively, the solver is consistent because of the equations (2.1)-(2.5), that are consistent with (1.6)-(1.10). The specific values used for $c, c_{a}$ do not play any role in this consistency. However, if we require the solver to have the highest accuracy, i.e. to be "tangent" to the original system, one has to take the speeds $c>c_{a}>0$ as approximations of $h \sqrt{a^{2}+g h}$ and $h|a|$ respectively, in the limit when $U_{l}, U_{r}$ are close to a common value $U$. This is because, as can be checked with straightforward computations, smooth solutions to (1.6)-(1.10) verify

$$
\begin{gathered}
\partial_{t}(h P)+\partial_{x}(h P u)+h^{2}\left(a^{2}+g h\right) \partial_{x} u=0, \\
\partial_{t}\left(h P_{\perp}\right)+\partial_{x}\left(h P_{\perp} u\right)+h^{2} a^{2} \partial_{x} v=0,
\end{gathered}
$$

that have to be compared with (2.6), (2.7). The accuracy of the solver on isolated contacts is described by the following lemma.

Lemma 2.1. The approximate Riemann solver $R\left(x / t, U_{l}, U_{r}\right)$ solves exactly:

(i) material contact discontinuities, 
(ii) left Alfven contact discontinuities under the condition $c_{a, l}=(h|a|)_{l}$,

(iii) right Alfven contact discontinuities under the condition $c_{a, r}=(h|a|)_{r}$.

Proof. Material contacts are solutions to the SWMHD system (1.6)-(1.10) with $u, v, P, P_{\perp}$ constant. These solutions are obviously solutions to the relaxation system (2.1)-(2.9) with $\pi=P, \pi_{\perp}=P_{\perp}$. Thus for these data, $R$ coincides with the exact solver, which proves (i).

Alfven contacts are solutions to (1.6)-(1.10) with $h, u, a, b \operatorname{sgn} a-v$ (for a left contact), $b \operatorname{sgn} a+v$ (for a right contact) constant. As previously, it is enough to prove that these solutions, completed with $\pi=P, \pi_{\perp}=P_{\perp}$, are solutions to the relaxation system (2.1)-(2.9). One can see that only (2.7) is not immediately satisfied. Comparing (2.7) to the second line of (2.11), we get the condition $c_{a}^{2}=h^{2} a^{2}$ where $v$ jumps. Note that according to (2.4) and (2.9), $h a$ and $c_{a}$ are both continuous through the Alfven waves (assuming $a \neq 0$ ). This yields (ii) and (iii).

Following the Godunov approach, the numerical scheme can be defined by the approximate Riemann solver as follows. We consider a mesh of cells $\left(x_{i-1 / 2}, x_{i+1 / 2}\right), i \in \mathbb{Z}$, of length $\Delta x_{i}=x_{i+1 / 2}-x_{i-1 / 2}$, discrete times $t_{n}$ with $t_{n+1}-t_{n}=\Delta t$, and cell values $U_{i}^{n}$ approximating the average of $U$ over the cell $i$ at time $t_{n}$. We can then define an approximate solution $U_{\text {appr }}(t, x)$ for $t_{n} \leq t<t_{n+1}$ and $x \in \mathbb{R}$ by

$$
U_{\text {appr }}(t, x)=R\left(\frac{x-x_{i+1 / 2}}{t-t_{n}}, U_{i}^{n}, U_{i+1}^{n}\right) \text { for } x_{i}<x<x_{i+1},
$$

where $x_{i}=\left(x_{i-1 / 2}+x_{i+1 / 2}\right) / 2$. This definition is coherent under a half CFL condition, formulated as

$$
\begin{aligned}
& x / t<-\frac{\Delta x_{i}}{2 \Delta t} \Rightarrow R\left(x / t, U_{i}, U_{i+1}\right)=U_{i}, \\
& x / t>\frac{\Delta x_{i+1}}{2 \Delta t} \Rightarrow R\left(x / t, U_{i}, U_{i+1}\right)=U_{i+1} .
\end{aligned}
$$

The new values at time $t_{n+1}$ are defined by

$$
U_{i}^{n+1}=\frac{1}{\Delta x_{i}} \int_{x_{i-1 / 2}}^{x_{i+1 / 2}} U_{a p p r}\left(t_{n+1}-0, x\right) d x .
$$

Notice that it is only in this averaging procedure that the choice of the particular pseudo-conservative variable $U$ as (1.13) is involved. We can follow the computations of [6, Section 2.3], the only difference being that the system is not conservative. We obtain the update formula

$$
U_{i}^{n+1}=U_{i}^{n}-\frac{\Delta t}{\Delta x_{i}}\left(\mathcal{F}_{l}\left(U_{i}^{n}, U_{i+1}^{n}\right)-\mathcal{F}_{r}\left(U_{i-1}^{n}, U_{i}^{n}\right)\right),
$$

where

$$
\begin{aligned}
& \mathcal{F}_{l}\left(U_{l}, U_{r}\right)=F\left(U_{l}\right)-\int_{-\infty}^{0}\left(R\left(\xi, U_{l}, U_{r}\right)-U_{l}\right) d \xi \\
& \mathcal{F}_{r}\left(U_{l}, U_{r}\right)=F\left(U_{r}\right)+\int_{0}^{\infty}\left(R\left(\xi, U_{l}, U_{r}\right)-U_{r}\right) d \xi,
\end{aligned}
$$


the variable $\xi$ stands for $x / t$, and the pseudo-conservative flux is chosen as

$$
F(U) \equiv\left(h u, h u^{2}+P, h u v+P_{\perp}, 0, h b u-h a v\right) .
$$

In (2.17), the two last components could be chosen differently since the two magnetic equations in our system are not conservative. We can remark that the choice of $F$ has no influence on the update formula (2.15).

\subsection{Energy inequality}

Here we do not use the entropy extension defined in [6, Definition 2.14], because the minimization principle is a bit too restrictive. We instead follow the strategy used in $[8,7]$. We define the left and right numerical energy fluxes as

$$
\begin{aligned}
& \mathcal{G}_{l}\left(U_{l}, U_{r}\right)=G\left(U_{l}\right)-\int_{-\infty}^{0}\left(E\left(R\left(\xi, U_{l}, U_{r}\right)\right)-E\left(U_{l}\right)\right) d \xi, \\
& \mathcal{G}_{r}\left(U_{l}, U_{r}\right)=G\left(U_{r}\right)+\int_{0}^{\infty}\left(E\left(R\left(\xi, U_{l}, U_{r}\right)\right)-E\left(U_{r}\right)\right) d \xi,
\end{aligned}
$$

where $E$ and $G$ are respectively the energy and the energy flux from (1.12),

$$
\begin{gathered}
E(U)=\frac{1}{2} h\left(u^{2}+v^{2}\right)+\frac{1}{2} g h^{2}+\frac{1}{2} h\left(a^{2}+b^{2}\right), \\
G(U)=\left(\frac{1}{2} h\left(u^{2}+v^{2}\right)+g h^{2}+\frac{1}{2} h\left(a^{2}+b^{2}\right)\right) u-h a(a u+b v) .
\end{gathered}
$$

Following [6, Section 2.3], a sufficient condition for the scheme to be energy satisfying is that

$$
\mathcal{G}_{r}\left(U_{l}, U_{r}\right)-\mathcal{G}_{l}\left(U_{l}, U_{r}\right) \leq 0 .
$$

When this is satisfied, because of the convexity of $E$ with respect to $U$ and of the CFL condition (2.13), one has the discrete energy inequality

$$
E\left(U_{i}^{n+1}\right)-E\left(U_{i}^{n}\right)+\frac{\Delta t}{\Delta x_{i}}\left(\mathcal{G}\left(U_{i}^{n}, U_{i+1}^{n}\right)-\mathcal{G}\left(U_{i-1}^{n}, U_{i}^{n}\right)\right) \leq 0,
$$

where the numerical energy flux $\mathcal{G}\left(U_{l}, U_{r}\right)$ is any function satisfying $\mathcal{G}_{r}\left(U_{l}, U_{r}\right) \leq$ $\mathcal{G}\left(U_{l}, U_{r}\right) \leq \mathcal{G}_{l}\left(U_{l}, U_{r}\right)$. In order to analyze the condition (2.20), let us introduce the sum of the gravitational potential energy and the magnetic energy

$$
e=\frac{1}{2} g h+\frac{1}{2}\left(a^{2}+b^{2}\right)
$$

that enables to rewrite the energy as

$$
E=\frac{1}{2} h\left(u^{2}+v^{2}\right)+h e .
$$

Then, while solving the relaxation system (2.1)-(2.9), we solve simultaneously the equation for a new variable $\hat{e}$,

$$
\partial_{t}\left(\hat{e}-\pi^{2} / 2 c^{2}-\pi_{\perp}^{2} / 2 c_{a}^{2}\right)+u \partial_{x}\left(\hat{e}-\pi^{2} / 2 c^{2}-\pi_{\perp}^{2} / 2 c_{a}^{2}\right)=0,
$$


where $\hat{e}$ has left and right initial data $e_{l}=e\left(U_{l}\right), e_{r}=e\left(U_{r}\right)$. The reason for writing (2.24) is that combined with (2.1)-(2.9) it implies

$$
\partial_{t}\left(\frac{1}{2} h\left(u^{2}+v^{2}\right)+h \hat{e}\right)+\partial_{x}\left(\left(\frac{1}{2} h\left(u^{2}+v^{2}\right)+h \hat{e}\right) u+\pi u+\pi_{\perp} v\right)=0 .
$$

Indeed, (2.25) can be obtained as follows. From (2.2), (2.3), (2.6), (2.7) (combined with (2.1)), we get

$$
\begin{gathered}
\partial_{t} u+u \partial_{x} u+\frac{1}{h} \partial_{x} \pi=0, \\
\partial_{t} v+u \partial_{x} v+\frac{1}{h} \partial_{x} \pi_{\perp}=0, \\
\partial_{t} \pi+u \partial_{x} \pi+\frac{c^{2}}{h} \partial_{x} u=0, \\
\partial_{t} \pi_{\perp}+u \partial_{x} \pi_{\perp}+\frac{c_{a}^{2}}{h} \partial_{x} v=0 .
\end{gathered}
$$

Multiplying these equations respectively by $u, v, \pi, \pi_{\perp}$, we obtain

$$
\begin{gathered}
\partial_{t} u^{2} / 2+u \partial_{x} u^{2} / 2+\frac{u}{h} \partial_{x} \pi=0, \\
\partial_{t} v^{2} / 2+u \partial_{x} v^{2} / 2+\frac{v}{h} \partial_{x} \pi_{\perp}=0, \\
\partial_{t} \pi^{2} / 2+u \partial_{x} \pi^{2} / 2+\frac{\pi c^{2}}{h} \partial_{x} u=0, \\
\partial_{t} \pi_{\perp}^{2} / 2+u \partial_{x} \pi_{\perp}^{2} / 2+\frac{\pi_{\perp} c_{a}^{2}}{h} \partial_{x} v=0 .
\end{gathered}
$$

Using the advection equations (2.8), (2.9) for $c$ and $c_{a}$, the two last equations of $(2.27)$ give

$$
\begin{gathered}
\partial_{t} \frac{\pi^{2}}{2 c^{2}}+u \partial_{x} \frac{\pi^{2}}{2 c^{2}}+\frac{\pi}{h} \partial_{x} u=0, \\
\partial_{t} \frac{\pi_{\perp}^{2}}{2 c_{a}^{2}}+u \partial_{x} \frac{\pi_{\perp}^{2}}{2 c_{a}^{2}}+\frac{\pi_{\perp}}{h} \partial_{x} v=0 .
\end{gathered}
$$

Adding up the two first equations of (2.27) and (2.28) yields

$\partial_{t}\left(\frac{u^{2}+v^{2}}{2}+\frac{\pi^{2}}{2 c^{2}}+\frac{\pi_{\perp}^{2}}{2 c_{a}^{2}}\right)+u \partial_{x}\left(\frac{u^{2}+v^{2}}{2}+\frac{\pi^{2}}{2 c^{2}}+\frac{\pi_{\perp}^{2}}{2 c_{a}^{2}}\right)+\frac{1}{h} \partial_{x}\left(\pi u+\pi_{\perp} v\right)=0$.

Then, adding this to (2.24) and using (2.1) finally gives our stated identity (2.25).

Using the value of the Riemann solution to the relaxation system at $x / t=0$, we define

$$
\mathcal{G}\left(U_{l}, U_{r}\right)=\left(\left(\frac{1}{2} h\left(u^{2}+v^{2}\right)+h \hat{e}\right) u+\pi u+\pi_{\perp} v\right)_{x / t=0} .
$$

Lemma 2.2. If for all values of $x / t$ the solution to (2.1)-(2.9) has nonnegative height $h$ and satisfies

$$
\hat{e} \geq e(U),
$$

where here $U=R\left(x / t, U_{l}, U_{r}\right), e(U)$ is defined in (2.22) and $\hat{e}$ is defined by (2.24), then $\mathcal{G}_{r}\left(U_{l}, U_{r}\right) \leq \mathcal{G}\left(U_{l}, U_{r}\right) \leq \mathcal{G}_{l}\left(U_{l}, U_{r}\right)$, and the discrete energy inequality (2.21) holds under the CFL condition (2.13). 
Proof. Since (2.25) is a conservative equation, and its conserved quantity and flux reduce to $E$ and $G$ on the left and right states, integrating it over rectangles one gets

$$
\begin{aligned}
\mathcal{G}\left(U_{l}, U_{r}\right) & =G\left(U_{l}\right)-\int_{-\infty}^{0}\left(\left(\frac{1}{2} h\left(u^{2}+v^{2}\right)+h \hat{e}\right)(\xi)-E\left(U_{l}\right)\right) d \xi \\
& =G\left(U_{r}\right)+\int_{0}^{\infty}\left(\left(\frac{1}{2} h\left(u^{2}+v^{2}\right)+h \hat{e}\right)(\xi)-E\left(U_{r}\right)\right) d \xi
\end{aligned}
$$

Therefore, comparing to (2.18), we see that in order to get $\mathcal{G}_{r} \leq \mathcal{G} \leq \mathcal{G}_{l}$ it is enough that for all $\xi$

$$
E\left(R\left(\xi, U_{l}, U_{r}\right)\right) \leq\left(\frac{1}{2} h\left(u^{2}+v^{2}\right)+h \hat{e}\right)(\xi),
$$

which is equivalent to (2.31).

\section{$2.3 \quad$ Intermediate states}

In this subsection we describe the solution to the Riemann problem for (2.1)(2.9) with initial data completed by the relations (2.10). The analysis is similar to that in $[8,9]$ for the full MHD system, and to [7] for shallow elastic fluids.

The quasilinear system (2.1)-(2.9) has the property of having a quasi diagonal form

$$
\begin{aligned}
\partial_{t}(\pi+c u)+(u+c / h) \partial_{x}(\pi+c u)-\frac{u}{h} c \partial_{x} c & =0, \\
\partial_{t}(\pi-c u)+(u-c / h) \partial_{x}(\pi-c u)-\frac{u}{h} c \partial_{x} c & =0, \\
\partial_{t}\left(1 / h+\pi / c^{2}\right)+u \partial_{x}\left(1 / h+\pi / c^{2}\right) & =0, \\
\partial_{t}\left(\pi_{\perp}+c_{a} v\right)+\left(u+c_{a} / h\right) \partial_{x}\left(\pi_{\perp}+c_{a} v\right)-\frac{v}{h} c_{a} \partial_{x} c_{a} & =0, \\
\partial_{t}\left(\pi_{\perp}-c_{a} v\right)+\left(u-c_{a} / h\right) \partial_{x}\left(\pi_{\perp}-c_{a} v\right)-\frac{v}{h} c_{a} \partial_{x} c_{a} & =0, \\
\partial_{t}\left(b+\frac{h a}{c_{a}^{2}} \pi_{\perp}\right)+u \partial_{x}\left(b+\frac{h a}{c_{a}^{2}} \pi_{\perp}\right) & =0, \\
\partial_{t}(h a)+u \partial_{x}(h a) & =0, \\
\partial_{t} c+u \partial_{x} c & =0, \\
\partial_{t} c_{a}+u \partial_{x} c_{a} & =0 .
\end{aligned}
$$

One deduces its eigenvalues, which are

$$
u-\frac{c}{h}, \quad u-\frac{c_{a}}{h}, \quad u, \quad u+\frac{c_{a}}{h}, \quad u+\frac{c}{h},
$$

the central eigenvalue $u$ having multiplicity 5 and the other being simple. From the above form one also checks easily that the system is hyperbolic, with all eigenvalues linearly degenerate. As a consequence, Rankine-Hugoniot conditions are well defined (the weak Riemann invariants do not jump through the associated discontinuity), and are equivalent to any conservative formulation. In the solution to the Riemann problem, the speeds corresponding to the previous eigenvalues will be denoted by

$$
\Sigma_{1}<\Sigma_{2}<\Sigma_{3}<\Sigma_{4}<\Sigma_{5},
$$


the speed $\Sigma_{3}$ corresponding to the eigenvalue $u$. Thus we get a 5 -wave solver with four intermediate states. The variables take the values "l" for $x / t<\Sigma_{1}$,

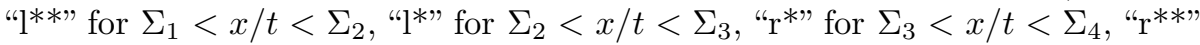
for $\Sigma_{4}<x / t<\Sigma_{5}$, "r" for $\Sigma_{5}<x / t$, see Figure 1. There are 5 strong Riemann

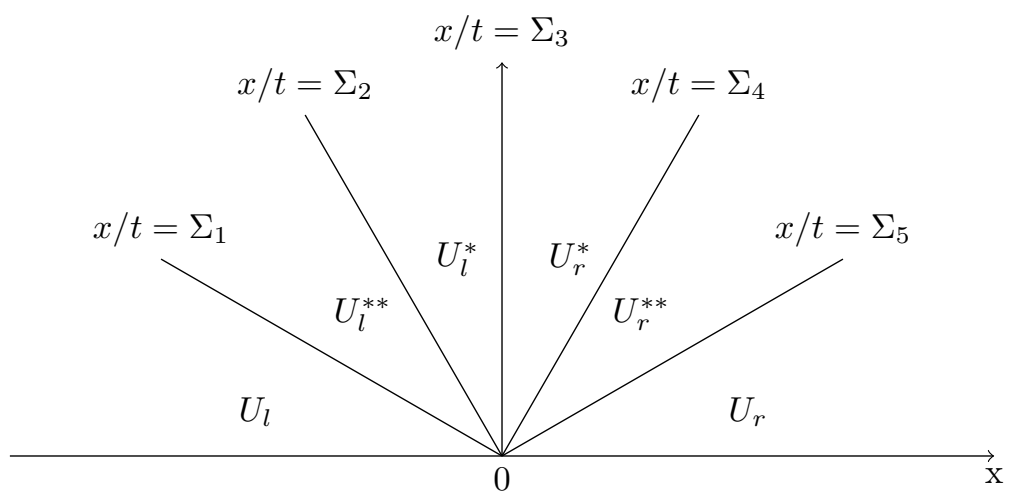

Figure 1: Intermediate states in the Riemann solution

invariants associated to the central wave (i.e. quantities that lie in the kernel of $\left.\partial_{t}+u \partial_{x}\right)$, which are

$$
c, \quad c_{a}, \quad h a, \quad \frac{1}{h}+\frac{\pi}{c^{2}}, \quad b+\frac{h a}{c_{a}^{2}} \pi_{\perp} .
$$

These quantities are thus weak Riemann invariants for the other waves. Four weak Riemann invariants for the central waves are $u, v, \pi, \pi_{\perp}$. They take the same value on the left and on the right of this central wave, we shall denote these values by $u^{*}, v^{*}, \pi^{*}, \pi_{\perp}^{*}$. The remaining weak Riemann invariants for the left and right waves are found to be

$$
\begin{array}{llll}
u-c / h: & \pi+c u, & \pi_{\perp}, & v, \\
u-c_{a} / h: & \pi_{\perp}+c_{a} v, & \pi, & u, \\
u+c_{a} / h: & \pi_{\perp}-c_{a} v, & \pi, & u, \\
u+c / h: & \pi-c u, & \pi_{\perp}, & v .
\end{array}
$$

We notice that the equations (2.1), (2.2), (2.6), (2.8) form a closed system of equations. Therefore, the variables $h, u, \pi, c$ can be resolved independently of the knowledge of $v, \pi_{\perp}, a, b, c_{a}$, and in particular they do not jump through the $\Sigma_{2}$ and $\Sigma_{4}$ waves. This means that for these unknowns $h, u, \pi, c$, the "l**" and "l*" states are identical, as well as the "r**" and "r*" states. In particular, $u$ and $\pi$ take the constant values $u^{*}$ and $\pi^{*}$ on the whole fan $\Sigma_{1}<x / t<\Sigma_{5}$,

$$
(u, \pi)_{l}^{* *}=(u, \pi)_{l}^{*}=(u, \pi)^{*}, \quad(u, \pi)_{r}^{* *}=(u, \pi)_{r}^{*}=(u, \pi)^{*} .
$$

The second velocity-pressure set of variables $v, \pi_{\perp}$ jump only though the $c_{a}$ waves $\Sigma_{2}, \Sigma_{4}$, thus

$$
\begin{gathered}
\left(v, \pi_{\perp}\right)_{l}^{* *}=\left(v, \pi_{\perp}\right)_{l}, \quad\left(v, \pi_{\perp}\right)_{r}^{* *}=\left(v, \pi_{\perp}\right)_{r}, \\
\left(v, \pi_{\perp}\right)_{l}^{*}=\left(v, \pi_{\perp}\right)_{r}^{*}=\left(v, \pi_{\perp}\right)^{*} .
\end{gathered}
$$


Then, because of (2.45), the variables $a, c_{a}$ do not jump through the $\Sigma_{2}$ and $\Sigma_{4}$ waves, as $h, u, \pi, c$. Moreover, $b$ does not jump through the $\Sigma_{1}$ and $\Sigma_{5}$ waves, as $v, \pi_{\perp}$, see Figure 2. In addition, computations using the Riemann invariants

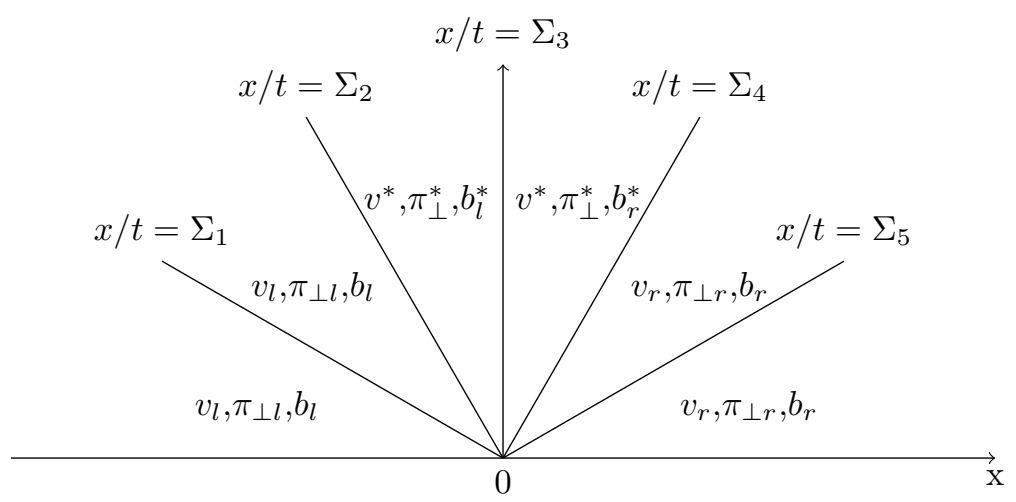

Figure 2: Intermediate states for the variable $v, \pi_{\perp}, b$

$(2.45),(2.46)$ give the values as in $[6,9]$

$$
\begin{aligned}
u^{*} & =\frac{c_{l} u_{l}+c_{r} u_{r}+\pi_{l}-\pi_{r}}{c_{l}+c_{r}}, \\
\pi^{*} & =\frac{c_{r} \pi_{l}+c_{l} \pi_{r}-c_{l} c_{r}\left(u_{r}-u_{l}\right)}{c_{l}+c_{r}}, \\
v^{*} & =\frac{c_{a l} v_{l}+c_{a r} v_{r}+\pi_{\perp l}-\pi_{\perp r}}{c_{a l}+c_{a r}}, \\
\pi_{\perp}^{*} & =\frac{c_{a r} \pi_{\perp l}+c_{a l} \pi_{\perp r}-c_{a l} c_{a r}\left(v_{r}-v_{l}\right)}{c_{a l}+c_{a r}},
\end{aligned}
$$

and

$$
\begin{aligned}
\frac{1}{h_{l}^{*}} & =\frac{1}{h_{l}}+\frac{c_{r}\left(u_{r}-u_{l}\right)+\pi_{l}-\pi_{r}}{c_{l}\left(c_{l}+c_{r}\right)}, \\
\frac{1}{h_{r}^{*}} & =\frac{1}{h_{r}}+\frac{c_{l}\left(u_{r}-u_{l}\right)+\pi_{r}-\pi_{l}}{c_{r}\left(c_{l}+c_{r}\right)} .
\end{aligned}
$$

Next, using the invariants in (2.45) that involve $a, b$, we get

$$
a_{l}^{*}=a_{l} \frac{h_{l}}{h_{l}^{*}}, \quad a_{r}^{*}=a_{r} \frac{h_{r}}{h_{r}^{*}},
$$

and

$$
\begin{aligned}
& b_{l}^{*}=b_{l}+\frac{h_{l} a_{l}}{c_{a l}\left(c_{a l}+c_{a r}\right)}\left(\pi_{\perp l}-\pi_{\perp r}+c_{a r}\left(v_{r}-v_{l}\right)\right), \\
& b_{r}^{*}=b_{r}+\frac{h_{r} a_{r}}{c_{a r}\left(c_{a l}+c_{a r}\right)}\left(\pi_{\perp r}-\pi_{\perp l}+c_{a l}\left(v_{r}-v_{l}\right)\right) .
\end{aligned}
$$

Using the previous formulas one can compute the speeds,

$$
\begin{gathered}
\Sigma_{1}=u_{l}-c_{l} / h_{l}, \quad \Sigma_{2}=u^{*}-c_{a l} / h_{l}^{*}, \quad \Sigma_{3}=u^{*}, \\
\Sigma_{4}=u^{*}+c_{a r} / h_{r}^{*}, \quad \Sigma_{5}=u_{r}+c_{r} / h_{r} .
\end{gathered}
$$


Finally, the intermediate values for $\hat{e}$ defined by (2.24) are given by

$$
\begin{aligned}
& \hat{e}_{l}^{*}=\frac{\left(\pi^{*}\right)^{2}}{2 c_{l}^{2}}+\frac{\left(\pi_{\perp}^{*}\right)^{2}}{2 c_{a l}^{2}}+e_{l}-\frac{\left(\pi_{l}\right)^{2}}{2 c_{l}^{2}}-\frac{\left(\pi_{\perp l}\right)^{2}}{2 c_{a l}^{2}}, \\
& \hat{e}_{l}^{* *}=\frac{\left(\pi^{*}\right)^{2}}{2 c_{l}^{2}}+\frac{\left(\pi_{\perp l}\right)^{2}}{2 c_{a l}^{2}}+e_{l}-\frac{\left(\pi_{l}\right)^{2}}{2 c_{l}^{2}}-\frac{\left(\pi_{\perp l}\right)^{2}}{2 c_{a l}^{2}}, \\
& \hat{e}_{r}^{*}=\frac{\left(\pi^{*}\right)^{2}}{2 c_{r}^{2}}+\frac{\left(\pi_{\perp}^{*}\right)^{2}}{2 c_{a r}^{2}}+e_{r}-\frac{\left(\pi_{r}\right)^{2}}{2 c_{r}^{2}}-\frac{\left(\pi_{\perp r}\right)^{2}}{2 c_{a r}^{2}}, \\
& \hat{e}_{r}^{* *}=\frac{\left(\pi^{*}\right)^{2}}{2 c_{r}^{2}}+\frac{\left(\pi_{\perp r}\right)^{2}}{2 c_{a r}^{2}}+e_{r}-\frac{\left(\pi_{r}\right)^{2}}{2 c_{r}^{2}}-\frac{\left(\pi_{\perp r}\right)^{2}}{2 c_{a r}^{2}},
\end{aligned}
$$

where $e_{l}, e_{r}$ stand for the values of $e$ defined by (2.22) on the left and right states respectively.

\section{Analysis of the energy inequality and choice of the speeds}

\subsection{Sufficient stability conditions for a fixed intermediate state}

In this subsection, we derive sufficient conditions for the inequality (2.31) in Lemma 2.2 to hold, for a fixed intermediate state

$$
U^{*}=\left(h^{*}, h^{*} u^{*}, h^{*} v^{*}, h^{*} a^{*}, h^{*} b^{*}\right),
$$

where the star '*' stands for any of "l**", "1*", "r*", "r**". These values are completed with those of the relaxation variables $\pi^{*}, \pi_{\perp}^{*}, c, c_{a}$, and of $\hat{e}^{*}$. Note that the notation ' $*$ ' differs here slightly from the one in the previous paragraph, in particular, $v^{*}$ and $\pi_{\perp}^{*}$ do not coincide with the values in (2.49) in case of the "l**" and " $\mathrm{r} * *$ " states. It is convenient to denote by the subscript ' $l / r$ ' any data evaluated on the initial state on the same side of the central wave as the intermediate state considered. The short notation $c, c_{a}$ will mean that these quantities are evaluated locally, i.e. at the ' $*$ ' state, or equivalently at the ' $l / r$ ' location since these quantities are strong invariants for the central wave.

In order to analyze (2.31), we use the same strategy as in [6, Lemma 2.20], and [8, Lemma 3.2], that consists in using a decomposition of $e\left(U^{*}\right)-\hat{e}^{*}$ in elementary entropy dissipation terms along each waves. This idea was introduced in [5] in the case of constant speeds.

Lemma 3.1. With the preceding notations, we have the identity

$$
\begin{aligned}
e\left(U^{*}\right)-\hat{e}^{*}= & D_{--}\left(U^{*}, u^{*}-\frac{\pi^{*}}{c}\right)+D_{-}\left(U^{*}, v^{*}-\frac{\pi_{\perp}^{*}}{c_{a}}\right) \\
& +D_{0}\left(U^{*}, U_{l / r}\right)+D_{+}\left(U^{*}, v^{*}+\frac{\pi_{\perp}}{c_{a}}\right)+D_{++}\left(U^{*}, u^{*}+\frac{\pi^{*}}{c}\right),
\end{aligned}
$$


where we set for any state $U$ and any scalar $\Lambda$, with $P$ and $P_{\perp}$ defined in (1.11),

$$
\begin{aligned}
D_{--}(U, \Lambda)= & \frac{1}{4}\left(u-\frac{P}{c}\right)^{2}-\frac{1}{4} \Lambda^{2} \\
& -(-P, u) \cdot\left(\frac{1}{2 c}\left(u-\frac{P}{c}-\Lambda\right), \frac{1}{2}\left(u-\frac{P}{c}-\Lambda\right)\right), \\
D_{++}(U, \Lambda)= & \frac{1}{4}\left(u+\frac{P}{c}\right)^{2}-\frac{1}{4} \Lambda^{2} \\
& -(-P, u) \cdot\left(-\frac{1}{2 c}\left(u+\frac{P}{c}-\Lambda\right), \frac{1}{2}\left(u+\frac{P}{c}-\Lambda\right)\right), \\
D_{-}(U, \Lambda)= & \frac{1}{4}\left(v-\frac{P_{\perp}}{c_{a}}\right)^{2}-\frac{1}{4} \Lambda^{2} \\
& -(b, v) \cdot\left(\frac{h a}{2 c_{a}}\left(v-\frac{P_{\perp}}{c_{a}}-\Lambda\right), \frac{1}{2}\left(v-\frac{P_{\perp}}{c_{a}}-\Lambda\right)\right), \\
D_{+}(U, \Lambda)= & \frac{1}{4}\left(v+\frac{P_{\perp}}{c_{a}}\right)^{2}-\frac{1}{4} \Lambda^{2} \\
& -(b, v) \cdot\left(-\frac{h a}{2 c_{a}}\left(v+\frac{P_{\perp}}{c_{a}}-\Lambda\right), \frac{1}{2}\left(v+\frac{P_{\perp}}{c_{a}}-\Lambda\right)\right), \\
& -\left(-P_{1}, b_{1}\right) \cdot\left(\begin{array}{l}
1 / h_{1}+P_{1} / c^{2}-1 / h_{2}-P_{2} / c^{2} \\
b_{1}+\frac{h_{1} a_{1}}{c_{a}^{2}} P_{1 \perp}-b_{2}-\frac{h_{2} a_{2}}{c_{a}^{2}} P_{2 \perp}
\end{array}\right) .
\end{aligned}
$$

Proof. We have to sum up the contributions in the right-hand side of (3.2). We look first at terms that are in factor of $P^{*} \equiv P\left(U^{*}\right)$,

$$
\begin{aligned}
\frac{1}{2 c}\left(u^{*}-\frac{P^{*}}{c}-u^{*}+\frac{\pi^{*}}{c}\right) & \text { in } D_{--}, \\
\frac{-1}{2 c}\left(u^{*}+\frac{P^{*}}{c}-u^{*}-\frac{\pi^{*}}{c}\right) & \text { in } D_{++}, \\
\left(\frac{1}{h^{*}}+\frac{P^{*}}{c^{2}}\right)-\left(\frac{1}{h_{l / r}}+\frac{P_{l / r}}{c^{2}}\right) & \text { in } D_{0} .
\end{aligned}
$$

Then, since $\frac{1}{h}+\frac{\pi}{c^{2}}$ is a strong invariant associated to the eigenvalue $u$,

$$
\frac{1}{h_{l / r}}+\frac{P_{l / r}}{c^{2}}=\frac{1}{h^{*}}+\frac{\pi^{*}}{c^{2}} .
$$

Thus the sum of (3.8), (3.9), (3.10) equals zero. Then, the terms in factor of $u^{*}$ in $D_{--}, D_{++}$also sum up to zero, as well as the terms in factor of $v^{*}$ in $D_{-}$, 
$D_{+}$. Then we look at the terms in factor of $-b^{*}$,

$$
\begin{aligned}
\frac{h^{*} a^{*}}{2 c_{a}}\left(v^{*}-\frac{P_{\perp}^{*}}{c_{a}}-v^{*}+\frac{\pi_{\perp}^{*}}{c_{a}}\right) & \text { in } D_{-}, \\
\frac{-h^{*} a^{*}}{2 c_{a}}\left(v^{*}+\frac{P_{\perp}^{*}}{c_{a}}-v^{*}-\frac{\pi_{\perp}^{*}}{c_{a}}\right) & \text { in } D_{+}, \\
\left(b^{*}+\frac{h^{*} a^{*}}{c_{a}^{2}} P_{\perp}^{*}\right)-\left(b_{l / r}+\frac{(h a)_{l / r}}{c_{a}^{2}} P_{\perp l / r}\right) & \text { in } D_{0} .
\end{aligned}
$$

But since $b+\frac{h a}{c_{a}^{2}} \pi_{\perp}$ is a strong invariant associated to the eigenvalue $u$, one has

$$
b_{l / r}+\frac{(h a)_{l / r}}{c_{a}^{2}} P_{\perp l / r}=b^{*}+\frac{h^{*} a^{*}}{c_{a}^{2}} \pi_{\perp}^{*} .
$$

Thus we get that the sum of (3.12), (3.13), (3.14) equals zero.

Now it remains to sum up the first lines from (3.3)-(3.7). Summing up the first lines from $D_{--}$and $D_{++}$we get

$$
\frac{1}{4}\left(u^{*}-\frac{P^{*}}{c}\right)^{2}-\frac{1}{4}\left(u^{*}-\frac{\pi^{*}}{c}\right)^{2}+\frac{1}{4}\left(u^{*}+\frac{P^{*}}{c}\right)^{2}-\frac{1}{4}\left(u^{*}+\frac{\pi^{*}}{c}\right)^{2}=\frac{\left(P^{*}\right)^{2}}{2 c^{2}}-\frac{\left(\pi^{*}\right)^{2}}{2 c^{2}} .
$$

Summing up the first lines from $D_{-}$and $D_{+}$we get

$$
\frac{1}{4}\left(v^{*}-\frac{P_{\perp}^{*}}{c_{a}}\right)^{2}-\frac{1}{4}\left(v^{*}-\frac{\pi_{\perp}^{*}}{c_{a}}\right)^{2}+\frac{1}{4}\left(v^{*}+\frac{P_{\perp}^{*}}{c_{a}}\right)^{2}-\frac{1}{4}\left(v^{*}+\frac{\pi_{\perp}^{*}}{c_{a}}\right)^{2}=\frac{\left(P_{\perp}^{*}\right)^{2}}{2 c_{a}^{2}}-\frac{\left(\pi_{\perp}^{*}\right)^{2}}{2 c_{a}^{2}} .
$$

The last terms are those from the first line of $D_{0}$,

$$
\left(e\left(U^{*}\right)-\frac{\left(P^{*}\right)^{2}}{2 c^{2}}-\frac{\left(P_{\perp}^{*}\right)^{2}}{2 c_{a}^{2}}\right)-\left(e(U)-\frac{P^{2}}{2 c^{2}}-\frac{P_{\perp}^{2}}{2 c_{a}^{2}}\right)_{l / r} .
$$

Moreover, according to $(2.24), \hat{e}-\frac{\pi^{2}}{2 c^{2}}-\frac{\pi_{\lrcorner}^{2}}{2 c_{a}^{2}}$ is a strong invariant associated to the eigenvalue $u$, which gives

$$
\left(e(U)-\frac{P^{2}}{2 c^{2}}-\frac{P_{\perp}^{2}}{2 c_{a}^{2}}\right)_{l / r}=\left(\hat{e}^{*}-\frac{\left(\pi^{*}\right)^{2}}{2 c^{2}}-\frac{\left(\pi_{\perp}^{*}\right)^{2}}{2 c_{a}^{2}}\right) .
$$

Summing up (3.16), (3.17) and (3.18), we get $e\left(U^{*}\right)-\hat{e}^{*}$, which proves the lemma.

We notice now that all dissipations excepted the central one in Lemma 3.1 can be written as opposite of squares,

$$
\begin{aligned}
& D_{--}(U, \Lambda)=-\frac{1}{4}\left(u-\frac{P}{c}-\Lambda\right)^{2}, D_{++}(U, \Lambda)=-\frac{1}{4}\left(u+\frac{P}{c}-\Lambda\right)^{2}, \\
& D_{-}(U, \Lambda)=-\frac{1}{4}\left(v-\frac{P_{\perp}}{c_{a}}-\Lambda\right)^{2}, D_{+}(U, \Lambda)=-\frac{1}{4}\left(v+\frac{P_{\perp}}{c_{a}}-\Lambda\right)^{2} .
\end{aligned}
$$

Thus they are all nonpositive, and in order to obtain a sufficient stability condition via Lemma 2.2 and Lemma 3.1, we need only to prove that $D_{0}$ from (3.7) is nonpositive. 
In order to analyze $D_{0}\left(U^{*}, U_{l / r}\right)$, we group the terms in factor of $1 / 2 c^{2}, 1 / 2 c_{a}^{2}$ and the terms where $b$ is involved, because they are squares, which gives using the expression of $P_{\perp}$ from (1.11) and the relation $h^{*} a^{*}=(h a)_{l / r}$,

$$
\begin{aligned}
D_{0}\left(U^{*}, U_{l / r}\right) & =\frac{g h^{*}}{2}-\frac{g h_{l / r}}{2}+\frac{\left(a^{*}\right)^{2}}{2}-\frac{a_{l / r}^{2}}{2}+P^{*}\left(\frac{1}{h^{*}}-\frac{1}{h_{l / r}}\right) \\
& +\frac{1}{2 c^{2}}\left(P^{*}-P_{l / r}\right)^{2}+\frac{1}{2 c_{a}^{2}}\left(P_{\perp}^{*}-P_{\perp l / r}\right)^{2}-\frac{1}{2}\left(b^{*}-b_{l / r}\right)^{2} .
\end{aligned}
$$

Next, we use the expression of $P$ and $P_{\perp}$ from (1.11) to obtain

$$
\begin{aligned}
D_{0}\left(U^{*}, U_{l / r}\right) & =\frac{g h^{*}}{2}-\frac{g h_{l / r}}{2}+\frac{g\left(h^{*}\right)^{2}}{2}\left(\frac{1}{h^{*}}-\frac{1}{h_{l / r}}\right) \\
& +\frac{\left(a^{*}\right)^{2}}{2}-\frac{a_{l / r}^{2}}{2}-h^{*}\left(a^{*}\right)^{2}\left(\frac{1}{h^{*}}-\frac{1}{h_{l / r}}\right) \\
& +\frac{1}{2 c^{2}}\left[\left(\frac{g\left(h^{*}\right)^{2}}{2}-\frac{g\left(h_{l / r}\right)^{2}}{2}\right)^{2}+(h a)_{l / r}^{2}\left(a^{*}-a_{l / r}\right)^{2}\right. \\
& \left.-2(h a)_{l / r}\left(a^{*}-a_{l / r}\right)\left(\frac{g\left(h^{*}\right)^{2}}{2}-\frac{g\left(h_{l / r}\right)^{2}}{2}\right)\right] \\
& -\frac{1}{2}\left(1-\frac{(h a)_{l / r}^{2}}{c_{a}^{2}}\right)\left(b^{*}-b_{l / r}\right)^{2}
\end{aligned}
$$

Obviously, under the condition $c_{a} \geq h_{l / r}\left|a_{l / r}\right|$, the last line is nonpositive. The following result is a particular case of [8, Lemma 3.3].

Lemma 3.2. For any $h_{*}>0, h_{l / r}>0$ one has

$$
\frac{g h_{*}}{2}-\frac{g h_{l / r}}{2}+\frac{g h_{*}^{2}}{2}\left(\frac{1}{h_{*}}-\frac{1}{h_{l / r}}\right)+\frac{1}{2} \frac{1}{\left(g h^{3}\right)_{*, l / r}}\left(\frac{g h_{*}^{2}}{2}-\frac{g h_{l / r}^{2}}{2}\right)^{2} \leq 0,
$$

with

$$
\left(g h^{3}\right)_{*, l / r}=\sup _{h \in\left[h_{l / r}, h_{*}\right]} g h^{3} .
$$

Using the inequality (3.24), we get an upper bound on the first line of the right-hand side of $(3.23)$,

$$
\begin{aligned}
D_{0}\left(U^{*}, U_{l / r}\right) \leq & -\frac{1}{2} \frac{1}{\left(g h^{3}\right)_{*, l / r}}\left(\frac{g\left(h^{*}\right)^{2}}{2}-\frac{g\left(h_{l / r}\right)^{2}}{2}\right)^{2} \\
& -\frac{(h a)_{l / r}^{2}}{2}\left(\frac{1}{h^{*}}-\frac{1}{h_{l / r}}\right)^{2} \\
& +\frac{1}{2 c^{2}}\left[\left(\frac{g\left(h^{*}\right)^{2}}{2}-\frac{g\left(h_{l / r}\right)^{2}}{2}\right)^{2}+(h a)_{l / r}^{4}\left(\frac{1}{h^{*}}-\frac{1}{h_{l / r}}\right)^{2}\right. \\
& \left.-2(h a)_{l / r}^{2}\left(\frac{1}{h^{*}}-\frac{1}{h_{l / r}}\right)\left(\frac{g\left(h^{*}\right)^{2}}{2}-\frac{g\left(h_{l / r}\right)^{2}}{2}\right)\right] .
\end{aligned}
$$


Finally, we can rewrite the right-hand side of (3.26) as a quadratic form,

$$
\begin{aligned}
D_{0}\left(U^{*}, U_{l / r}\right) \leq & -\left(\frac{1}{2\left(g h^{3}\right)_{*, l / r}}-\frac{1}{2 c^{2}}\right)\left(\frac{g\left(h^{*}\right)^{2}}{2}-\frac{g\left(h_{l / r}\right)^{2}}{2}\right)^{2} \\
& -\frac{1}{2}(h a)_{l / r}^{2}\left(1-\frac{(h a)_{l / r}^{2}}{c^{2}}\right)\left(\frac{1}{h^{*}}-\frac{1}{h_{l / r}}\right)^{2} \\
& -\frac{(h a)_{l / r}^{2}}{c^{2}}\left(\frac{1}{h^{*}}-\frac{1}{h_{l / r}}\right)\left(\frac{g\left(h^{*}\right)^{2}}{2}-\frac{g\left(h_{l / r}\right)^{2}}{2}\right) .
\end{aligned}
$$

It leads to the following proposition stating that the entropy condition reduces to subcharacteristic conditions.

Proposition 3.3. In order to have $e\left(U^{*}\right)-\hat{e}^{*} \leq 0$ at the intermediate state $U^{*}$ (ensuring the discrete energy inequality according to Lemma 2.2), it is enough that $h^{*}>0$ and

$$
(h a)_{l / r}^{2}+\left(g h^{3}\right)_{*, l / r} \leq c^{2}
$$

and

$$
(h a)_{l / r}^{2} \leq c_{a}^{2},
$$

where $\left(g h^{3}\right)_{*, l / r}$ is defined in (3.25), and $(h a)_{l / r}, c, c_{a}$ are evaluated on the same side of the central wave as is $U^{*}$.

Proof. We use Lemma 3.1 and the previous analysis. With the condition (3.29), it yields (3.27). It is now sufficient that the right-hand side in (3.27) is a non positive quadratic form. Thus it is sufficient that

$$
\left(\frac{(h a)_{l / r}^{2}}{c^{2}}\right)^{2} \leq(h a)_{l / r}^{2}\left(1-\frac{(h a)_{l / r}^{2}}{c^{2}}\right)\left(\frac{1}{\left(g h^{3}\right)_{*, l / r}}-\frac{1}{c^{2}}\right),
$$

or

$$
\frac{(h a)_{l / r}^{2}}{c^{2}} \leq\left(1-\frac{(h a)_{l / r}^{2}}{c^{2}}\right)\left(\frac{c^{2}}{\left(g h^{3}\right)_{*, l / r}}-1\right) .
$$

Developing the right-hand side and simplifying, we obtain

$$
\frac{(h a)_{l / r}^{2}}{\left(g h^{3}\right)_{*, l / r}} \leq \frac{c^{2}}{\left(g h^{3}\right)_{*, l / r}}-1 .
$$

Multiplying by $\left(g h^{3}\right)_{*, l / r}$ we get (3.28), which concludes the proof.

\subsection{Choice of signal speeds}

In this subsection we derive explicit values for the signal speeds $c, c_{a}$ that are sufficient for having positivity of height and (3.28), (3.29), that yield the energy inequality. Such values have been found in [6, Proposition 2.18] for Euler equations and in [9] for MHD equations. We use here the approach of [9], that enables to treat negative pressures $\pi$. We make the following a priori choice of the relaxation speeds $c_{l}, c_{r}$,

$$
\begin{aligned}
& c_{l}=h_{l} s_{l}+\frac{3}{2} h_{l}\left(\left(u_{l}-u_{r}\right)_{+}+\frac{\left(\pi_{r}-\pi_{l}\right)_{+}}{h_{l} s_{l}+h_{r} s_{r}}\right), \\
& c_{r}=h_{r} s_{r}+\frac{3}{2} h_{r}\left(\left(u_{l}-u_{r}\right)_{+}+\frac{\left(\pi_{l}-\pi_{r}\right)_{+}}{h_{l} s_{l}+h_{r} s_{r}}\right),
\end{aligned}
$$


with

$$
s=\sqrt{a^{2}+g h} .
$$

This choice for $c_{l}$ and $c_{r}$ implies in particular that $c_{l} \geq h_{l} s_{l}$ and $c_{r} \geq h_{r} s_{r}$. We set

$$
X_{l}=\frac{1}{s_{l}}\left(\left(u_{l}-u_{r}\right)_{+}+\frac{\left(\pi_{r}-\pi_{l}\right)_{+}}{h_{l} s_{l}+h_{r} s_{r}}\right),
$$

so that by (3.33), one has

$$
\frac{c_{l}}{h_{l}}=s_{l}\left(1+\frac{3}{2} X_{l}\right) .
$$

Now we observe that we have two intermediate states on the left, but they have the same density $h_{l}^{*}$. Thus the conditions (3.28), (3.29) are identical for the "l**" and "l*" states, and refer to the height $h_{l}^{*}$. We estimate it from (2.50),

$$
\begin{aligned}
\frac{1}{h_{l}^{*}} & =\frac{1}{h_{l}}+\frac{c_{r}\left(u_{r}-u_{l}\right)+\pi_{l}-\pi_{r}}{c_{l}\left(c_{l}+c_{r}\right)} \\
& \geq \frac{1}{h_{l}}-\frac{c_{r}\left(u_{l}-u_{r}\right)_{+}}{c_{l}\left(c_{l}+c_{r}\right)}-\frac{\left(\pi_{r}-\pi_{l}\right)_{+}}{c_{l}\left(c_{l}+c_{r}\right)} \\
& \geq \frac{1}{h_{l}}-\frac{\left(u_{l}-u_{r}\right)_{+}}{c_{l}}-\frac{\left(\pi_{r}-\pi_{l}\right)_{+}}{c_{l}\left(h_{l} s_{l}+h_{r} s_{r}\right)} .
\end{aligned}
$$

Using (3.35) and (3.36), one gets

$$
\frac{1}{h_{l}^{*}} \geq \frac{1}{h_{l}}\left(1-\frac{X_{l}}{1+\frac{3}{2} X_{l}}\right) .
$$

Thus $h_{l}^{*}>0$, and

$$
0<h_{l}^{*} \leq h_{l} / x_{l},
$$

with

$$
x_{l}=1-\frac{X_{l}}{1+\frac{3}{2} X_{l}} \in(1 / 3,1] .
$$

This allows to estimate the supremum in (3.25),

$$
\sqrt{\left(g h^{3}\right)_{*, l / r}} \leq \frac{h_{l}}{x_{l}} \sqrt{\frac{g h_{l}}{x_{l}}} .
$$

We can estimate the right-hand side using the following result from [9, Lemma 3.1].

Lemma 3.4. Consider a pressure law $p(\rho)$ defined for $\rho>0$, satisfying

$$
\begin{gathered}
\frac{d}{d \rho}\left(\rho \sqrt{p^{\prime}}\right)>0 \\
\frac{d}{d \rho}\left(\rho \sqrt{p^{\prime}}\right) \leq \alpha \sqrt{p^{\prime}}, \quad \text { for some constant } \alpha>1 .
\end{gathered}
$$

Let $x=1-X /(1+\alpha X)$ for some $X \geq 0$. Then for all $\rho>0$,

$$
\frac{\rho}{x} \sqrt{p^{\prime}\left(\frac{\rho}{x}\right)} \leq \rho \sqrt{p^{\prime}(\rho)}(1+\alpha X) .
$$


The assumptions concerning the pressure (3.44)-(3.45) are satisfied with $p(\rho)=g \rho^{2} / 2$ and $\alpha=3 / 2$. Therefore we can apply this result with $\rho=h_{l}$, $X=X_{l}$, which gives

$$
\frac{h_{l}}{x_{l}} \sqrt{\frac{g h_{l}}{x_{l}}} \leq h_{l} \sqrt{g h_{l}}\left(1+\frac{3}{2} X_{l}\right)
$$

Thus, using (3.43) and (3.47), for getting (3.28) it is enough that

$$
(h a)_{l}^{2}+g h_{l}^{3}\left(1+\frac{3}{2} X_{l}\right)^{2} \leq c_{l}^{2}
$$

But using (3.36) and the definition of $s_{l}$ from (3.34),

$$
\begin{aligned}
c_{l}^{2} & =s_{l}^{2} h_{l}^{2}\left(1+\frac{3}{2} X_{l}\right)^{2} \\
& =(h a)_{l}^{2}\left(1+\frac{3}{2} X_{l}\right)^{2}+g h_{l}^{3}\left(1+\frac{3}{2} X_{l}\right)^{2},
\end{aligned}
$$

which yields (3.48). The same analysis is valid on the right, with

$$
X_{r}=\frac{1}{s_{r}}\left(\left(u_{l}-u_{r}\right)_{+}+\frac{\left(\pi_{l}-\pi_{r}\right)_{+}}{h_{l} s_{l}+h_{r} s_{r}}\right), \quad x_{r}=1-\frac{X_{r}}{1+\frac{3}{2} X_{r}} .
$$

Proposition 3.5. The solver is positive in height and entropy satisfying for the choice of $c_{l}, c_{r}$ given by (3.33) and $c_{a l}, c_{a r}$ given by

$$
c_{a l}=(h|a|)_{l}, \quad c_{a r}=(h|a|)_{r} .
$$

Proof. We apply Proposition 3.3. The above computations show that $h_{l}^{*}, h_{r}^{*}>0$ and that (3.28) holds. The choice (3.52) gives obviously (3.29).

Lemma 3.6 (Bounds on the propagation speeds). The formulas (3.33) ensure the following estimate on propagation speeds:

$$
\max \left(\frac{c_{l}}{h_{l}}, \frac{c_{r}}{h_{r}}\right) \leq C\left(\left(u_{l}-u_{r}\right)_{+}+s_{l}+s_{r}\right),
$$

where $C>0$ is an absolute constant.

Proof. We have $|P| \leq h s^{2}$ with $P=g \frac{h^{2}}{2}-h a^{2}$ and $s^{2}=a^{2}+g h$. Since $\pi_{l}=P_{l}$ and $\pi_{r}=P_{r}$, the result follows obviously.

\section{Properties of the relaxation approximate Rie- mann solver}

Before stating our main result, we have to explicit the numerical fluxes and the CFL condition. The solution to the Riemann problem for the relaxation system (2.1)-(2.9) has five wave speeds $\Sigma_{1}<\Sigma_{2}<\Sigma_{3}<\Sigma_{4}<\Sigma_{5}$, that can be computed by $(2.53)$. The intermediate states $l^{* *}, l^{*}, r^{*}, r^{* *}$ have been determined 
in Section 2.3. We would like now to compute the left/right numerical fluxes $\mathcal{F}_{l}, \mathcal{F}_{r}$ that are involved in the update formula (2.15).

All components of the system except $h a$ and $h b$ are conservative, thus classical computations give the associated numerical fluxes,

$$
\mathcal{F}_{l}=\left(\mathcal{F}^{h}, \mathcal{F}^{h u}, \mathcal{F}^{h v}, \mathcal{F}_{l}^{h a}, \mathcal{F}_{l}^{h b}\right), \quad \mathcal{F}_{r}=\left(\mathcal{F}^{h}, \mathcal{F}^{h u}, \mathcal{F}^{h v}, \mathcal{F}_{r}^{h a}, \mathcal{F}_{r}^{h b}\right),
$$

where the conservative part involves the Riemann solution evaluated at $\mathrm{x} / \mathrm{t}=0$,

$$
\begin{aligned}
& \mathcal{F}^{h}=(h u)_{x / t=0}, \\
& \mathcal{F}^{h u}=\left(h u^{2}+\pi\right)_{x / t=0}, \\
& \mathcal{F}^{h v}=\left(h u v+\pi_{\perp}\right)_{x / t=0} .
\end{aligned}
$$

More explicitly (4.2) yields that the quantities between parentheses are evaluated at "l" if $\Sigma_{1} \geq 0$, at " $l^{* *}$ " if $\Sigma_{1} \leq 0 \leq \Sigma_{2}$, at "l*" if $\Sigma_{2} \leq 0 \leq \Sigma_{3}$, at " $r^{*}$ " if $\Sigma_{3} \leq 0 \leq \Sigma_{4}$, at " $r^{* *}$ " if $\Sigma_{4} \leq 0 \leq \Sigma_{5}$, at " $r$ " if $\Sigma_{5} \leq 0$. As usual there is no ambiguity when equality occurs in these conditions. Similarly, the numerical energy flux is computed according to (2.30).

We complete these formulas by computing the left/right numerical fluxes for the variables $h a$ and $h b$ from (2.16),

$$
\begin{gathered}
\mathcal{F}_{l}^{h a}=0+\min \left(0, \Sigma_{3}\right)\left((h a)_{r}-(h a)_{l}\right), \\
\mathcal{F}_{r}^{h a}=0-\max \left(0, \Sigma_{3}\right)\left((h a)_{r}-(h a)_{l}\right), \\
\mathcal{F}_{l}^{h b}=(h b u-h a v)_{l}+\min \left(0, \Sigma_{1}\right)\left((h b)_{l}^{* *}-(h b)_{l}\right) \\
+\min \left(0, \Sigma_{2}\right)\left((h b)_{l}^{*}-(h b)_{l}^{* *}\right)+\min \left(0, \Sigma_{3}\right)\left((h b)_{r}^{*}-(h b)_{l}^{*}\right) \\
+\min \left(0, \Sigma_{4}\right)\left((h b)_{r}^{* *}-(h b)_{r}^{*}\right)+\min \left(0, \Sigma_{5}\right)\left((h b)_{r}-(h b)_{r}^{* *}\right), \\
\mathcal{F}_{r}^{h b}=(h b u-h a v)_{r}-\max \left(0, \Sigma_{1}\right)\left((h b)_{l}^{* *}-(h b)_{l}\right) \\
-\max \left(0, \Sigma_{2}\right)\left((h b)_{l}^{*}-(h b)_{l}^{* *}\right)-\max \left(0, \Sigma_{3}\right)\left((h b)_{r}^{*}-(h b)_{l}^{*}\right) \\
-\max \left(0, \Sigma_{4}\right)\left((h b)_{r}^{* *}-(h b)_{r}^{*}\right)-\max \left(0, \Sigma_{5}\right)\left((h b)_{r}-(h b)_{r}^{* *}\right) .
\end{gathered}
$$

Using the computation performed in [9, Subsection 5.3], these fluxes can be also written

$$
\begin{aligned}
& \text { If } \Sigma_{3} \geq 0 \text { then }\left\{\begin{array}{l}
\mathcal{F}_{l}^{h b}=(h b u-h a v)_{x / t=0-}, \\
\mathcal{F}_{r}^{h b}=(h b u-h a v)_{x / t=0-}-v^{*}\left((h a)_{r}-(h a)_{l}\right),
\end{array}\right. \\
& \text { If } \Sigma_{3} \leq 0 \text { then }\left\{\begin{array}{l}
\mathcal{F}_{l}^{h b}=(h b u-h a v)_{x / t=0+}+v^{*}\left((h a)_{r}-(h a)_{l}\right), \\
\mathcal{F}_{r}^{h b}=(h b u-h a v)_{x / t=0+},
\end{array}\right.
\end{aligned}
$$

where $v^{*}$ is the central value of $v$ defined in (2.49) (and indeed $\Sigma_{3}=u^{*}$ ).

The maximal propagation speed is then

$$
A\left(U_{l}, U_{r}\right)=\max \left(\left|\Sigma_{1}\right|,\left|\Sigma_{2}\right|,\left|\Sigma_{3}\right|,\left|\Sigma_{4}\right|,\left|\Sigma_{5}\right|\right)=\max \left(\left|\Sigma_{1}\right|,\left|\Sigma_{5}\right|\right) .
$$

The CFL condition (2.13) becomes

$$
\Delta t A\left(U_{i}, U_{i+1}\right) \leq \frac{1}{2} \min \left(\Delta x_{i}, \Delta x_{i+1}\right) .
$$


Note that with (3.53) and (2.53) we get

$$
A\left(U_{l}, U_{r}\right) \leq C\left(\left|u_{l}\right|+\left|u_{r}\right|+s_{l}+s_{r}\right)
$$

with $C$ an absolute constant, bounding the propagation speed of the approximate Riemann solver by the left and right true speeds. This property is also valid in [7] for shallow water elastic fluids and is more general than the possibility of treating data with vacuum considered in [6]. Note that for getting (4.10), no restriction on the ratio $h_{l} / h_{r}$ is required.

We are now able to obtain our main result.

Theorem 4.1. Assume that the initial data $U_{l}, U_{r}$ satisfy $h_{l}>0, h_{r}>0$ and use the choice of the speeds (3.33), (3.52) with $\pi_{l}=P\left(U_{l}\right), \pi_{r}=P\left(U_{r}\right)$. Under the CFL condition (4.8), (4.9), the Riemann solver defined by the intermediate states and speeds $\Sigma_{i}$ computed in Subsection 2.3 and the numerical fluxes $\mathcal{F}_{l}\left(U_{l}, U_{r}\right), \mathcal{F}_{r}\left(U_{l}, U_{r}\right)$ defined via (4.1)-(4.7), has the following properties.

(i) It keeps the positivity of $h$,

(ii) The approximate Riemann solver satisfies the discrete energy inequality (2.21), with numerical energy flux (2.30),

(iii) Isolated material contact discontinuities are resolved exactly,

(iv) Isolated Alfven contact discontinuities are resolved exactly,

(v) Resonant material-Alfven contact discontinuities defined by

$$
h=c s t, u=c s t, a=0,
$$

are resolved exactly,

(vi) Data with bounded propagation speeds give finite numerical propagation speed, according to (4.10),

(vii) The numerical viscosity is sharp, in the sense that the propagation speeds $\Sigma_{i}$ of the approximate Riemann solver tend to the exact propagation speeds when the left and right states $U_{l}, U_{r}$ tend to a common value,

(viii) The variables $h, h u, h v$ are conservative, and the scheme satisfies an asymptotic consistency with the non-conservative part of the system for smooth data.

Proof. The items (i), (ii) are consequences of Proposition 3.5 and Lemma 2.2. The items (iii), (iv) hold according to Lemma 2.1. The proof of (v) is straightforward with the formulas of Section 2.3, since in this case $\Sigma_{2}=\Sigma_{3}=\Sigma_{4}$. The item (vi) has been proved with Lemma 3.6. The item (vii) is obvious with (3.33), (3.52). The property that $h, h u, h v$ are conservative is also obvious.

Thus it remains to prove the statement of (viii) concerning the consistency. Denote as in the introduction $\mathbf{b}=(a, b), \mathbf{u}=(u, v)$. Then the magnetic equations of the original system (1.9), (1.10) can be written

$$
\partial_{t}(h \mathbf{b})+\partial_{x}(h \mathbf{b} u-h a \mathbf{u})+\mathbf{u} \partial_{x}(h a)=0 .
$$


At the discrete level, the $h \mathbf{b}$ components of the update formula (2.15) involve the numerical fluxes from (4.1)

$$
\mathcal{F}_{l}^{h \mathbf{b}} \equiv\left(\mathcal{F}_{l}^{h a}, \mathcal{F}_{l}^{h b}\right), \quad \mathcal{F}_{r}^{h \mathbf{b}} \equiv\left(\mathcal{F}_{r}^{h a}, \mathcal{F}_{r}^{h b}\right) .
$$

Using the formulas for the fluxes (4.3) and (4.6), (4.7), we observe that

$$
\mathcal{F}_{r}^{h \mathbf{b}}-\mathcal{F}_{l}^{h \mathbf{b}}=-\mathbf{u}^{*}\left((h a)_{r}-(h a)_{l}\right) .
$$

Thus, asymptotically when $U_{l}, U_{r} \rightarrow U$, one has

$$
\mathcal{F}_{r}^{h \mathbf{b}}\left(U_{l}, U_{r}\right)-\mathcal{F}_{l}^{h \mathbf{b}}\left(U_{l}, U_{r}\right)=-\mathbf{u}\left((h a)_{r}-(h a)_{l}\right)+o\left(\left|U_{l}-U\right|+\left|U_{r}-U\right|\right) .
$$

Since obviously $\mathcal{F}_{l}^{h \mathbf{b}}(U, U)=\mathcal{F}_{r}^{h \mathbf{b}}(U, U)=h \mathbf{b} u$ - hau, we conclude the asymptotic consistency with (4.12) for smooth solutions (in the sense proposed in [6, Section 4.2]), which concludes the proof of (viii).

\section{$5 \quad$ Numerical tests}

In this section we perform numerical computations in order to evaluate the properties of the scheme, in relation with Theorem 4.1. First and second order methods in time and space are evaluated, the latter using an ENO reconstruction, as described in [6, section 4.13]. The conservative variable is $U$ as in (1.13), and the slope limitations are performed on the variables $h, u, v, h a, b$.

We take 200 points, and plot a reference solution obtained by a first order computation with 10000 points. The CFL-number is taken $1 / 2$ in all tests. The space variable $x$ is taken in $[0,1], g=9.81$. Neumann boundary conditions are applied. Three test cases are investigated:

- Test 1 is a Riemann problem with $(h a)_{l}=(h a)_{r}$,

- Test 2 is a generic Riemann problem with positive height,

- Test 3 is a Riemann problem where the initial heights are taken positive on the left side and vanishing on the right side.

The numerical values for Test 1 are given in Table 1 . In this case, $h a=1 / 2$ remains constant, in accordance with (1.9). The first order method in time and space is evaluated, with our numerical fluxes defined by (4.1)-(4.7). Note that ha remains stricly constant at the discrete level, because of (4.3). Our results are compared to those obtained with the HLL flux, see for example [6, Equation (2.111)], applied here to the system (1.6)-(1.8), (1.10) with $h a=1 / 2$, which is conservative. We ommit to plot the $h a$ component, since it is constant in both methods. The reference solution is plotted in Figure 3. It consists of, from left to right, a left rarefaction wave, a left Alfven contact, a right Alfven contact, a right shock. There is no material contact. We observe as expected that the components $h, u$ only vary through fast waves whereas the components $v$ and $b$ only vary through Alfven waves. In Figure 4 we observe that the performances on fast waves are extremely similar for both methods, whereas for Alfven waves, our 5 -wave solver shows a much better resolution than the 2-wave HLL solver. 
The numerical values for Test 2 are given in Table 2 . The reference solution is plotted in Figure 5. It consists of, from left to right, a left rarefaction wave, a left Alfven contact, a material contact, a right Alfven contact, and a right shock. We observe in Figure 6 that the second order resolution improves the sharpness of contact discontinuities, but sometimes gives rise to slight instabilities.

The numerical values for Test 3 are given in Table 3 . The reference solution is plotted in Figure 7. It consists of, from left to right, a left rarefaction wave, a left Alfven contact, a right rarefaction wave. There is no material contact, nor right Alfven contact. The numerical results are shown in Figure 8. We notice that on the right the height $h$ vanishes, and the variables $u, v, a, b$ take eventually non-physical values, due to the fact that only the conservative variables $h u, h v, h a, h b$ make sense. Taking this into account, we observe that the computed solution achieves a good accuracy.

\begin{tabular}{|c|ccccc|}
\hline Values of $x$ & $h$ & $u$ & $v$ & $a$ & $b$ \\
\hline $\mathrm{x} \leq 0.5$ & 1. & 0.2 & 0.7 & 0.5 & 0.4 \\
\hline $0.5<\mathrm{x} \leq 1$ & 0.5 & -0.1 & 0.3 & 1. & 0.1 \\
\hline
\end{tabular}

Table 1: Initial data for Test 1 , the Riemann problem with $h a=1 / 2$

\begin{tabular}{|c|ccccc|}
\hline Values of $x$ & $h$ & $u$ & $v$ & $a$ & $b$ \\
\hline $\mathrm{x} \leq 0.5$ & 1.4 & 0.2 & 0.6 & 1 & 0.4 \\
\hline $0.5<\mathrm{x} \leq 1$ & 0.2 & -0.1 & 0.3 & 1.2 & 0.1 \\
\hline
\end{tabular}

Table 2: Initial data for Test 2, the Riemann problem with positive height

\begin{tabular}{|c|ccccc|}
\hline Values of $x$ & $h$ & $u$ & $v$ & $a$ & $b$ \\
\hline $\mathrm{x} \leq 0.5$ & 2. & 1. & 2.5 & 0.8 & 0.4 \\
\hline $0.5<\mathrm{x} \leq 1$ & 0. & 0. & 0. & 0. & 0. \\
\hline
\end{tabular}

Table 3: Initial data for Test 3, the Riemann problem with vanishing height 


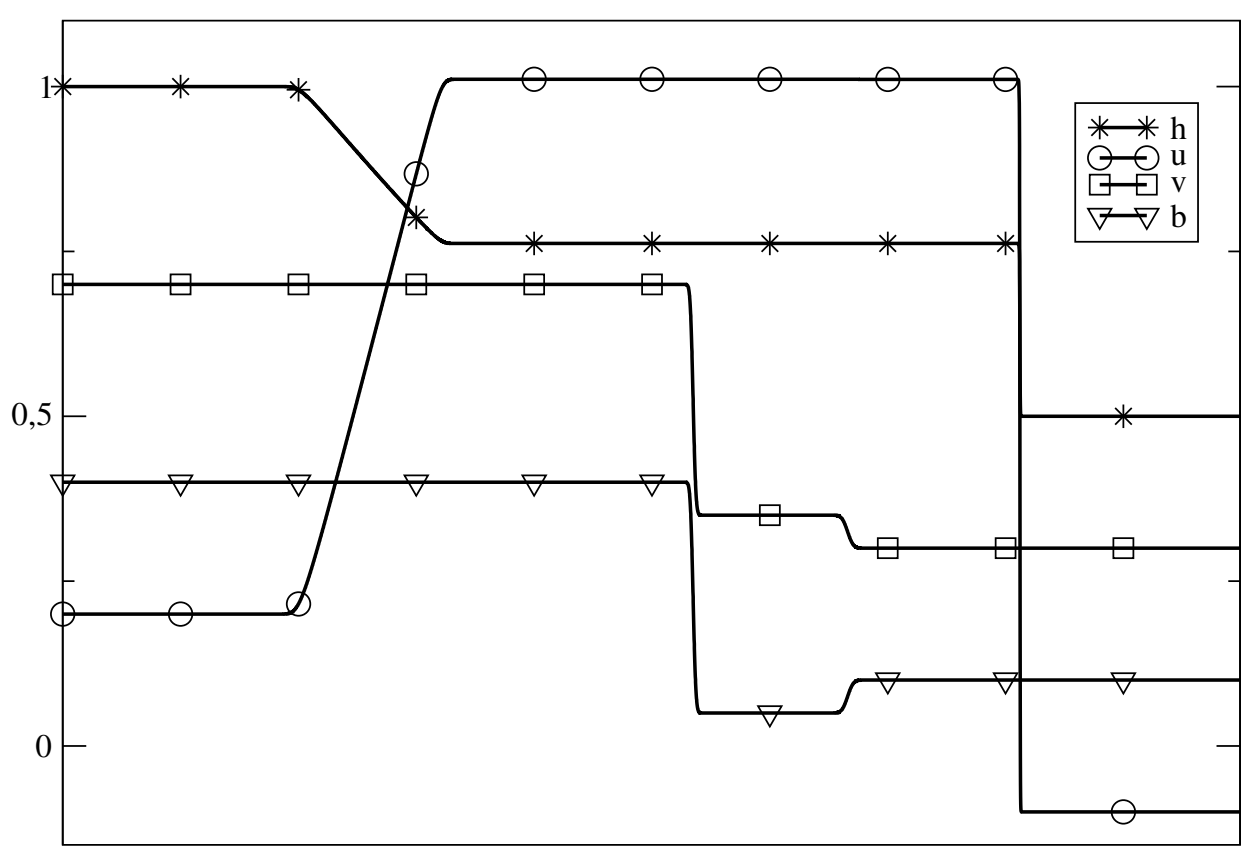

Figure 3: Riemann solution for Test 1 at time $t=0.1$ computed with the HLL flux or the 5-wave solver with 10000 points 

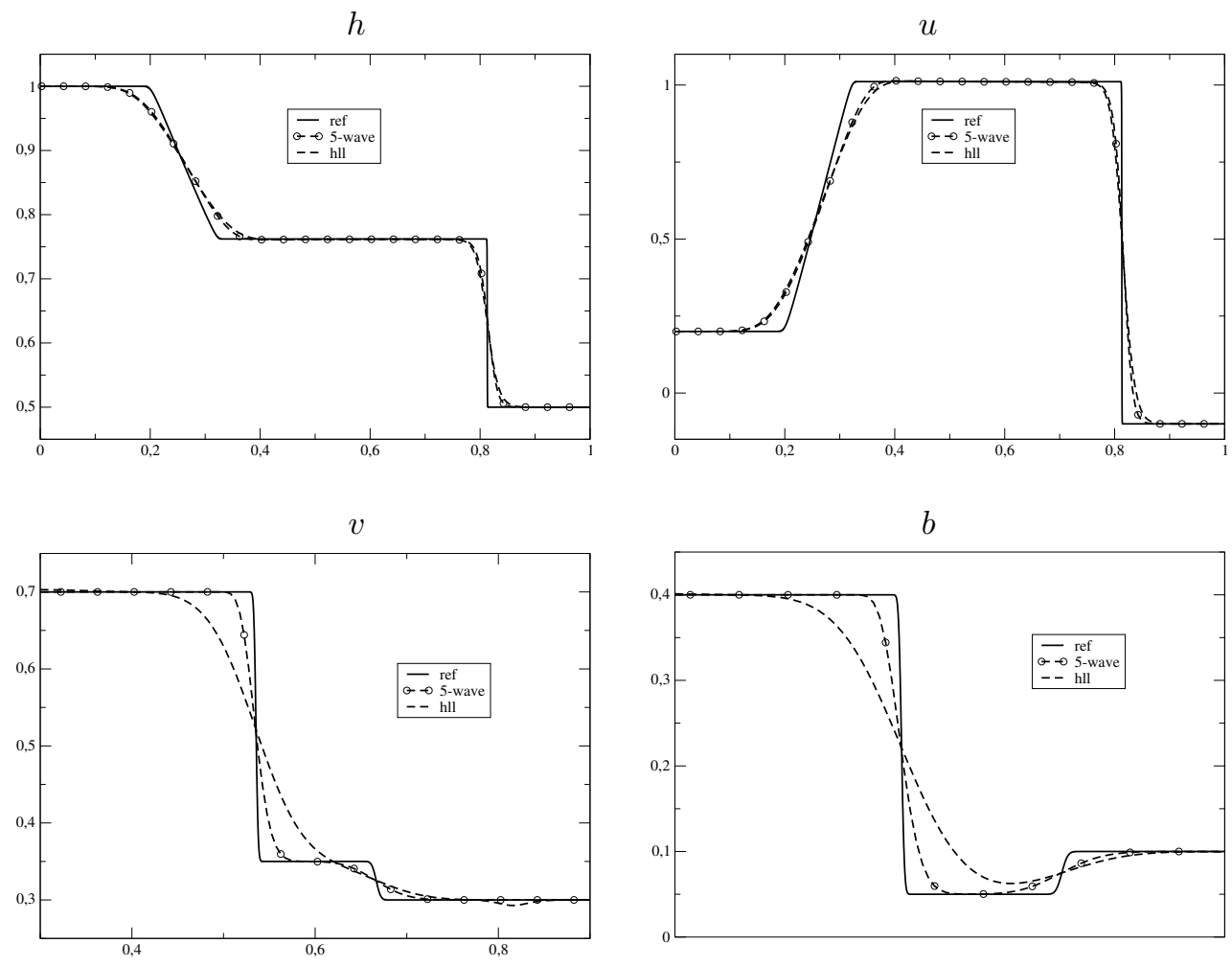

Figure 4: Components $h, u, v, b$ for Test 1 at time $t=0.1$ computed with the HLL flux and the 5 -wave solver with 200 points. The reference solution is the continuous line. 


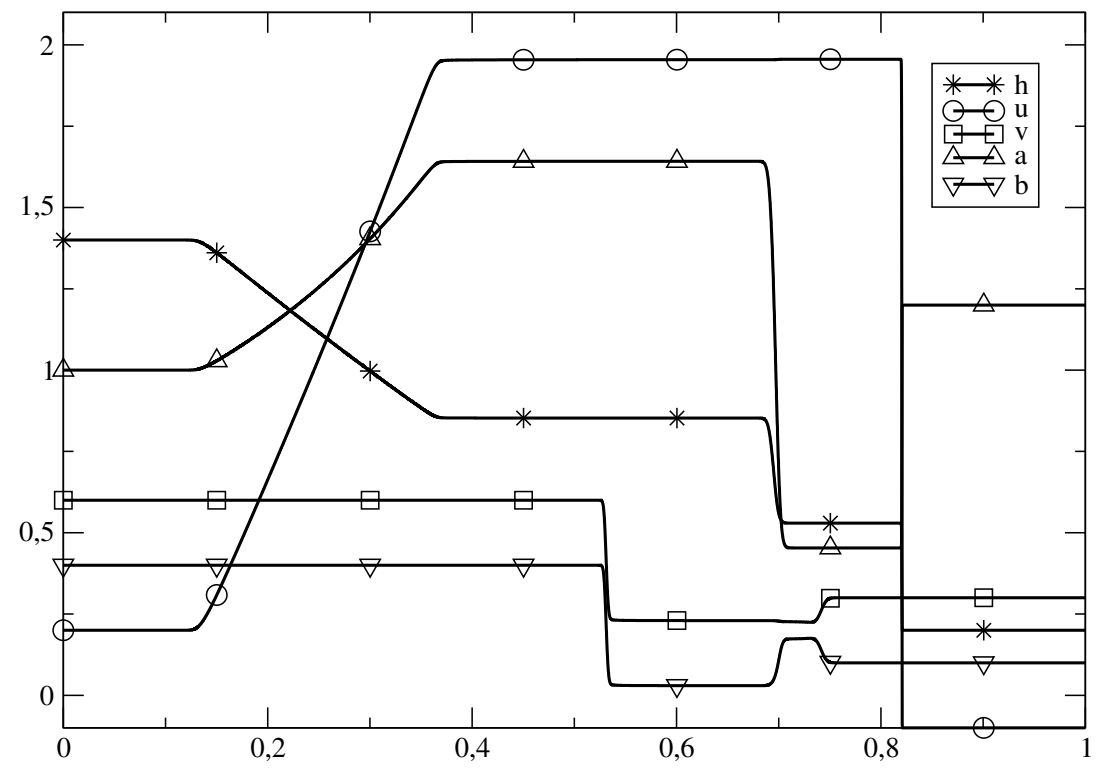

Figure 5: Reference solution for Test 2 at time $t=0.1$ computed at first order with 10000 points 

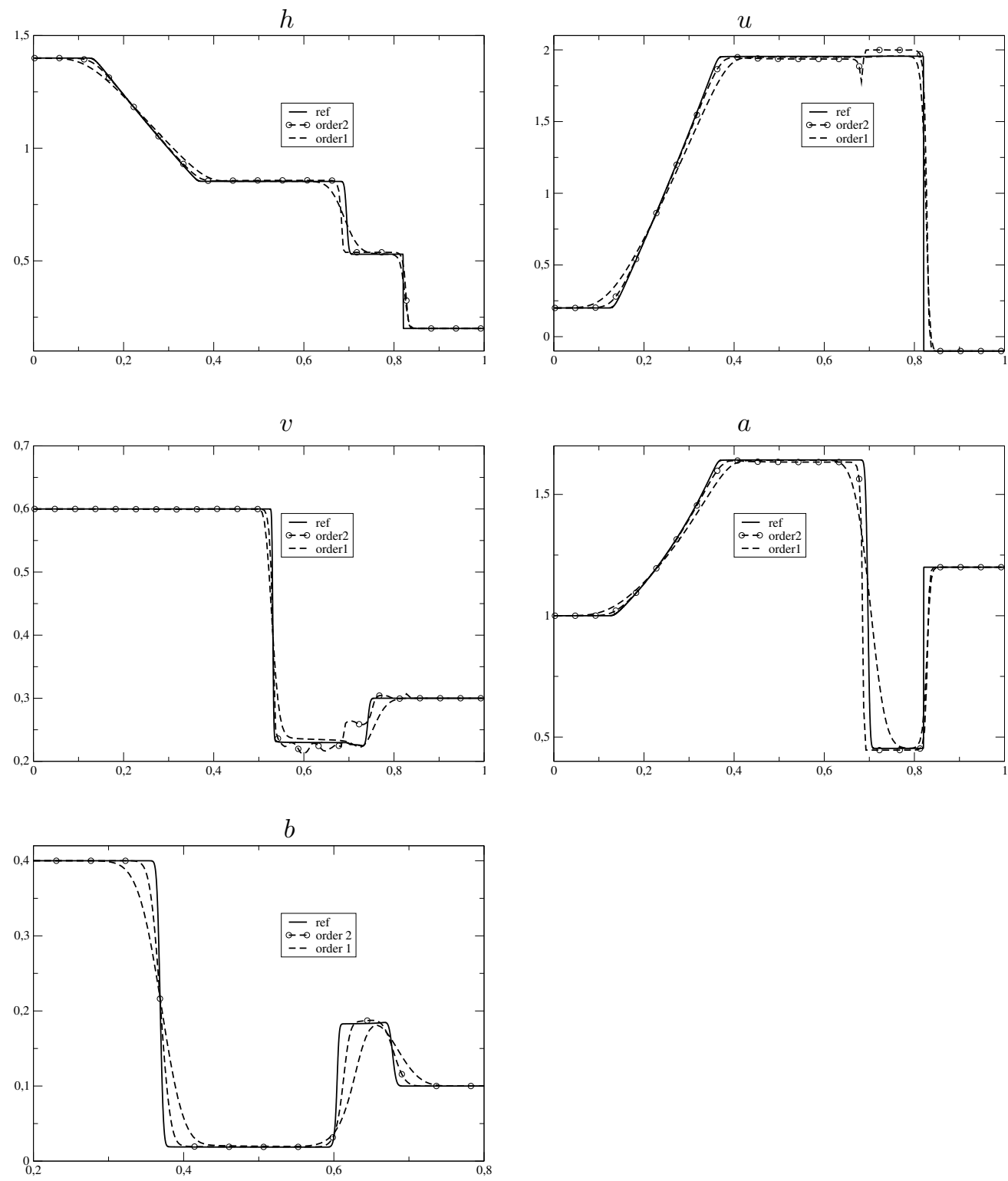

Figure 6: Components $h, u, v, a, b$ for Test 2 at time $t=0.1$ computed at first and second order with 200 points. The reference solution is the continuous line. 


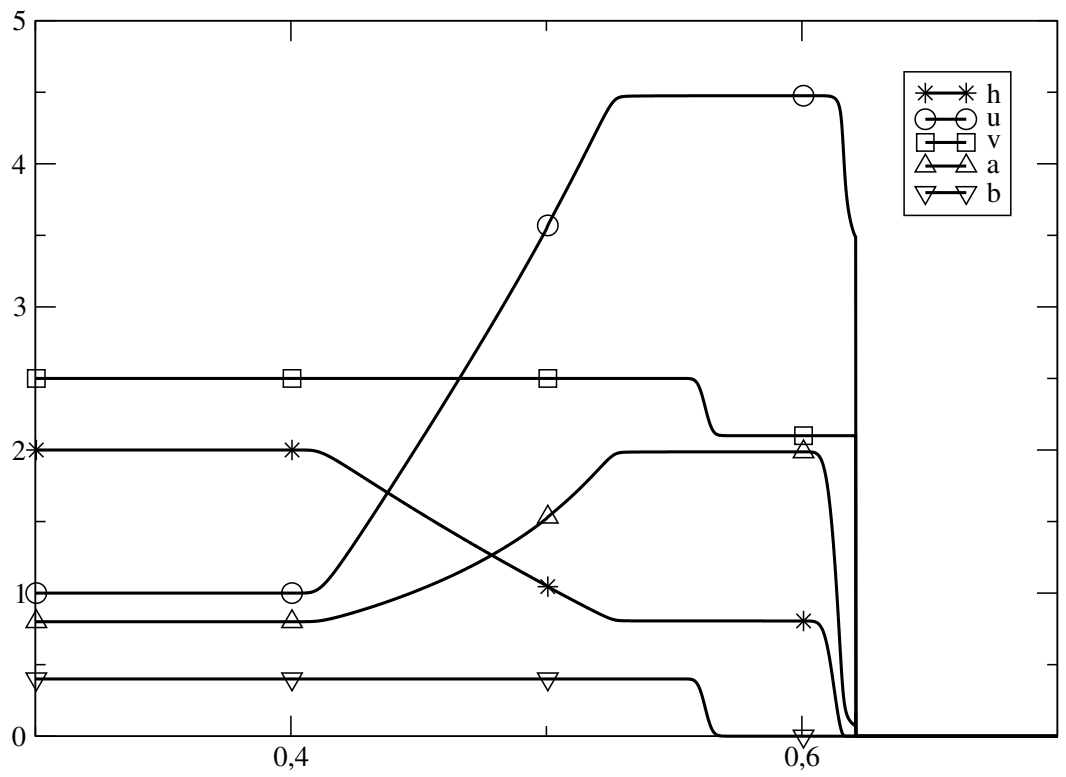

Figure 7: Reference solution for Test 3 at time $t=0.05$ computed at first order with 10000 points 

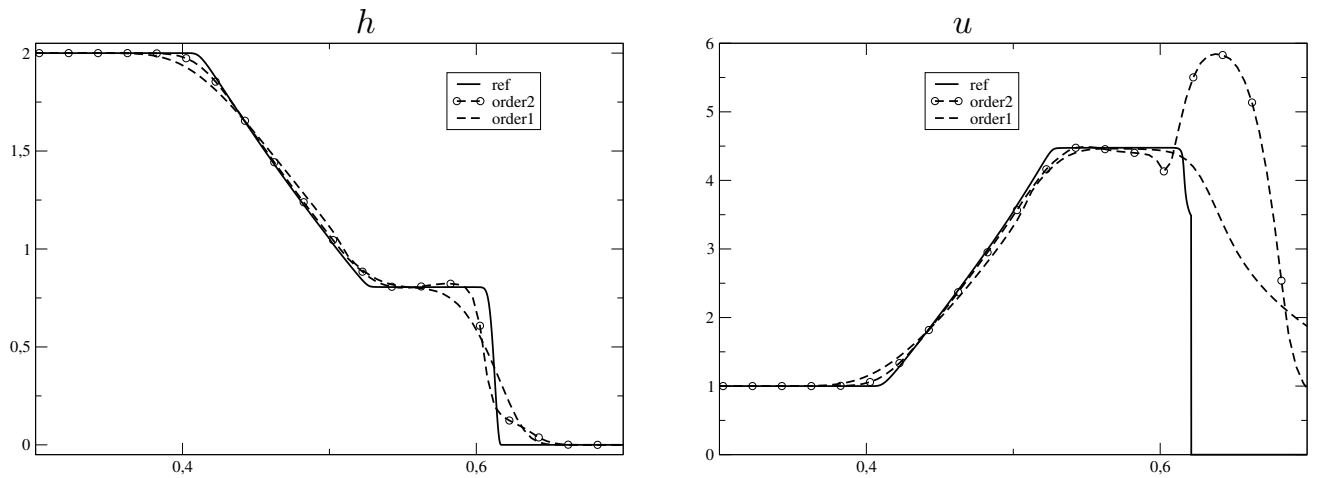

$v$

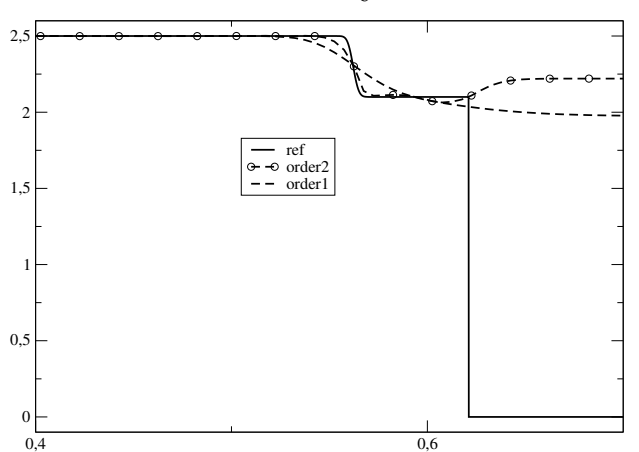

a

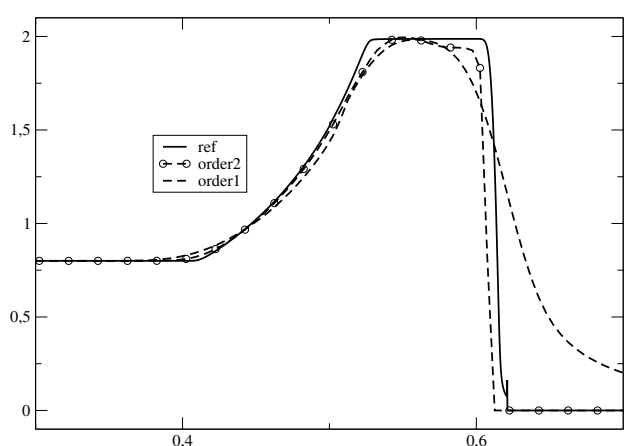

b

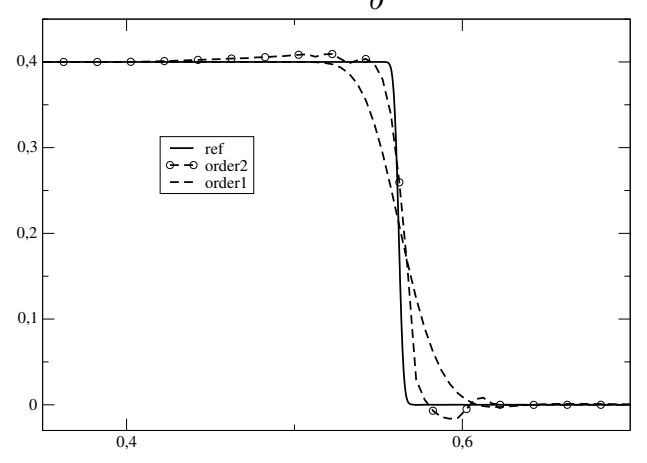

Figure 8: Components $h, u, v, a, b$ for Test 3 at time $t=0.05$ computed at first and second order with 200 points. The reference solution is the continuous line. The height $h$ vanishes on the right state, leading to irrelevant values of $u, v, a$, $b$ since only the conservative variables $h u, h v, h a, h b$ make sense. 


\section{References}

[1] D.S. Balsara, M. Dumbser, R. Abgrall, Multidimensional HLL and HLLC Riemann solvers for unstructured meshes with application to Euler and MHD flows, J. Comput. Phys. 261 (2014), 172-208.

[2] C. Berthon, P. Charrier, B. Dubroca, An HLLC scheme to solve the M1 model of radiative transfer in two space dimensions, J. Sci. Comput. 31 (2007), 347-389.

[3] C. Berthon, B. Dubroca, A. Sangam, A local entropy minimum principle for deriving entropy preserving schemes, SIAM Numer. Anal. 50 (2012), 468-491.

[4] F. Bézard, B. Després, An entropic solver for ideal Lagrangian magnetohydrodynamics, J. Comput. Phys. 154 (1999), 65-89.

[5] F. Bouchut, Entropy satisfying flux vector splittings and kinetic BGK models, Numer. Math. 94 (2003), 623-672.

[6] F. Bouchut, Nonlinear stability of finite volume methods for hyperbolic conservation laws, and well-balanced schemes for sources, Birkhäuser, 2004.

[7] F. Bouchut, S. Boyaval, A new model for shallow viscoelastic fluids, Math. Models and Meth. in Appl. Sci. 23 (2013), 1479-1526.

[8] F. Bouchut, C. Klingenberg, K. Waagan, A multiwave approximate Riemann solver for ideal MHD based on relaxation I - Theoretical framework, Numer. Math. 108 (2007), 7-42.

[9] F. Bouchut, C. Klingenberg, K. Waagan, A multiwave approximate Riemann solver for ideal MHD based on relaxation II - Numerical implementation with 3 and 5 waves, Numer. Math. 115 (2010), 647-679.

[10] C. Chalons, F. Coquel, C. Marmignon, Well-balanced time implicit formulation of relaxation schemes for the Euler equations, SIAM J. Sci. Comput. 30 (2007), 394-415.

[11] C. Chalons, F. Coquel, Modified Suliciu relaxation system and exact resolution of isolated shock waves, Math. Models Methods Appl. Sci. 24 (2014), 937-971.

[12] F. Coquel, E. Godlewski, N. Seguin, Relaxation of fluid systems, Math. Models Methods Appl. Sci. 22 (2012), 1250014.

[13] P.J. Dellar, Hamiltonian and symmetric hyperbolic structures of shallow water magnetohydrodynamics, Physics of Plasmas 9 (2002), 1130.

[14] B. Després, Lagrangian systems of conservation laws. Invariance properties of Lagrangian systems of conservation laws, approximate Riemann solvers and the entropy condition, Numer. Math. 89 (2001), 99-134.

[15] H. DeSterck, Hyperbolic theory of the shallow water magnetohydrodynamics equations, Physics of Plasmas 8 (2001), 3293 
[16] F.G. Fuchs, A.D. McMurry, S. Mishra, N.H. Risebro, K. Waagan, Approximate Riemann solvers and robust high-order finite volume schemes for multi-dimensional ideal MHD equations, Comm. Comput. Phys. 9 (2011), 324-362.

[17] G. Gallice, Positive and entropy stable Godunov-type schemes for gas dynamics and MHD equations in Lagrangian or Eulerian coordinates, Numer. Math. 94 (2003), 673-713.

[18] P.A. Gilman, Magnetohydrodynamic "shallow water" equations for the solar tachocline, Astrophys. J. Lett. 544 (2000), L79-L82.

[19] A. Harten, P.D. Lax, B. Van Leer, On upstream differencing and Godunovtype schemes for hyperbolic conservation laws, SIAM Review 25 (1983), $35-61$.

[20] P. Janhunen, A Positive conservative method for magnetohydrodynamics based on HLL and Roe methods, J. Comput. Phys. 160 (2000), 649-661.

[21] J.A. Rossmanith, A constrained transport method for the shallow water MHD equations, in Hyperbolic problems: theory, numerics, applications, 9th International Conference on Hyperbolic Problems, Pasadena, 2002, Ed. T.Y. Hou, E. Tadmor, 851-860, 2003.

[22] J.A. Rossmanith, D.S. Bale, R.J. Leveque, A wave propagation algorithm for hyperbolic systems on curved manifolds, J. Comput. Phys. 199 (2004), 631-662.

[23] A. Sangam, An HLLC scheme for ten-moments approximation coupled with magnetic field, Int. J. Comput. Sci. Math. 2 (2008), 73-109.

[24] I. Suliciu, Some stability-instability problems in phase transitions modelled by piecewise linear elastic or viscoelastic constitutive equations, Internat. J. Engrg. Sci. 30 (1992), 483-494.

[25] K. Waagan, C. Federrath, C. Klingenberg, A robust numerical scheme for highly compressible magnetohydrodynamics: Nonlinear stability, implementation and tests, J. Comput. Phys. 230 (2011) 3331-3351.

[26] V. Zeitlin, Remarks on rotating shallow-water magnetohydrodynamics, Nonlinear Processes in Geophysics 20 (2013), 893-898. 